\title{
ON THE KINEMATIC SEPARATION OF FIELD AND CLUSTER STARS ACROSS THE BULGE GLOBULAR NGC 6528
}

\author{
E. P. Lagioia ${ }^{1}$, A. P. Milone ${ }^{2,3,4}$, P. B. Stetson ${ }^{5}$, G. Bono ${ }^{1,6}$, P. G. Prada Moroni ${ }^{7}$, \\ M. Dall'Ora ${ }^{8}$, A. Aparicio ${ }^{3,4}$, R. Buonanno ${ }^{1,9}$, A. CAlamida $^{6,10}$, I. Ferraro $^{6}$, R. Gilmozzi $^{11}$, \\ G. IAnNicola ${ }^{6}$, N. MAtsunaga ${ }^{12}$, M. Monelli ${ }^{3,4}$, AND A. Walker ${ }^{13}$ \\ ${ }_{1}^{1}$ Dipartimento di Fisica, Università degli Studi di Roma-Tor Vergata, via della Ricerca Scientifica 1, I-00133 Roma, Italy; eplagioia@ roma2.infn.it \\ ${ }^{2}$ Research School of Astronomy and Astrophysics, The Australian National University, Cotter Road, Weston, ACT 2611, Australia \\ ${ }^{3}$ Instituto de Astrofisica de Canarias, E-38200 La Laguna, Tenerife, Canary Islands, Spain \\ ${ }^{4}$ Department of Astrophysics, University of La Laguna. E-38200 La Laguna, Tenerife, Canary Islands, Spain \\ ${ }^{5}$ Dominion Astrophysical Observatory, Herzberg Institute of Astrophysics, National Research Council, 5071 West Saanich Road, Victoria, BC V9E 2E7, Canada \\ ${ }^{6}$ INAF-Osservatorio Astronomico di Roma, Via Frascati 33, I-00044 Monte Porzio Catone, Italy \\ ${ }^{7}$ Dipartimento di Fisica, Università di Pisa, I-56127 Pisa, Italy \\ ${ }^{8}$ INAF-Osservatorio Astronomico di Capodimonte, Salita Moiariello 16, I-80131 Napoli, Italy \\ ${ }^{9}$ INAF-Osservatorio Astronomico Collurania, via M. Maggini, I-64100 Teramo, Italy \\ ${ }^{10}$ Space Telescope Science Institute, 3700 San Martin Drive, Baltimore, MD 21218, USA \\ ${ }^{11}$ European Southern Observatory, Karl-Schwarzschild-Straße 2, D-85748 Garching, Germany \\ 12 Kiso Observatory, Institute of Astronomy, School of Science, The University of Tokyo, 10762-30, Mitake, Kiso-machi, Kiso-gun, 3 Nagano 97-0101, Japan \\ ${ }^{13}$ Cerro Tololo Inter-American Observatory, National Optical Astronomy Observatory, Casilla 603, La Serena, Chile \\ Received 2013 April 24; accepted 2013 December 4; published 2014 January 27
}

\begin{abstract}
We present deep and precise multi-band photometry of the Galactic bulge globular cluster NGC 6528. The current data set includes optical and near-infrared images collected with ACS/WFC, WFC3/UVIS, and WFC3/IR on board the Hubble Space Telescope. The images cover a time interval of almost $10 \mathrm{yr}$, and we have been able to carry out a proper-motion separation between cluster and field stars. We performed a detailed comparison in the $m_{\mathrm{F} 814 \mathrm{~W}}, m_{\mathrm{F} 606 \mathrm{~W}}-m_{\mathrm{F} 814 \mathrm{~W}}$ color-magnitude diagram with two empirical calibrators observed in the same bands. We found that NGC 6528 is coeval with and more metal-rich than 47 Tuc. Moreover, it appears older and more metalpoor than the super-metal-rich open cluster NGC 6791. The current evidence is supported by several diagnostics (red horizontal branch, red giant branch bump, shape of the sub-giant branch, slope of the main sequence) that are minimally affected by uncertainties in reddening and distance. We fit the optical observations with theoretical isochrones based on a scaled-solar chemical mixture and found an age of $11 \pm 1 \mathrm{Gyr}$ and an iron abundance slightly above solar $([\mathrm{Fe} / \mathrm{H}]=+0.20)$. The iron abundance and the old cluster age further support the recent spectroscopic findings suggesting a rapid chemical enrichment of the Galactic bulge.
\end{abstract}

Key words: globular clusters: general - globular clusters: individual (NGC 6528, NGC 104, NGC 6791) stars: evolution

Online-only material: color figures

\section{INTRODUCTION}

In one of the three seminal papers given by Baade (1958) at the famous Vatican conference held in Rome in 1957, he discussed the stellar populations in the Galactic bulge. It is interesting to note that Baade called the same region van Tulder's pole. van Tulder (1942), a few years before, published an interesting paper concerning the location of the Galactic pole by using different types of stars and open clusters. Thus, we suggest that this low-reddening region should be defined as the van Tulder-Baade's window.

This region is dominated by the presence of two Galactic globular clusters (GGCs), namely, NGC 6528 and NGC 6522. The former is a very interesting cluster, since it is among the most metal-rich GGCs. Spectroscopic investigations, based on high-resolution spectra, suggest for this cluster a solar metallicity and a modest $\alpha$-element enhancement. Carretta et al. (2001), studying four red horizontal branch (HB) stars, found $[\mathrm{Fe} / \mathrm{H}]=+0.07 \pm 0.01$ and a marginal $\alpha$ enhancement $([\alpha / \mathrm{Fe}] \approx+0.2)$, while Zoccali et al. (2004) investigated three giants belonging to $\mathrm{HB}$ and to the red giant branch (RGB) and found $[\mathrm{Fe} / \mathrm{H}]=-0.1 \pm 0.2$ and $[\alpha / \mathrm{Fe}] \approx+0.1$. In a similar investigation, based on high-resolution near-infrared (NIR) spectra of four bright giants, Origlia et al. (2005) found
$[\mathrm{Fe} / \mathrm{H}]=-0.17 \pm 0.01$ and $[\alpha / \mathrm{Fe}]=+0.33 \pm 0.01$. The above measurements indicate that NGC 6528 is an ideal laboratory to constrain the possible occurrence of an age-metallicity relation among the most metal-rich and old GGCs (Rakos \& Schombert 2005; Dotter et al. 2011). Moreover, NGC 6528 and its twin NGC 6553 are considered the prototype metal-rich clusters of the Galactic bulge (Ortolani et al. 1995; Zoccali et al. 2001) and therefore, unique stellar systems to constrain the difference between cluster and field stars.

Estimates of both structural parameters and intrinsic properties for NGC 6528 are partially plagued by the high differential reddening across the field of view (FOV) and by the strong level of contamination from bulge and disk stars.

The effect of differential reddening and field-star contamination are among the reasons why age estimates for NGC 6528 range from $13 \pm 2 \mathrm{Gyr}$ (Ortolani et al. 2001; based on isochrones provided by Cassisi \& Salaris 1997 and Castellani et. al 1999) to $11 \pm 2$ Gyr (Feltzing \& Johnson 2002; isochrones by Salasnich et al. 2000), to 12.6 Gyr (Momany et al. 2003; isochrones by Bertelli et al. 1994).

In principle, the combination of visual and NIR photometry is an efficient diagnostic to separate candidate cluster and field stars (Calamida et al. 2009; Bono et al. 2010). Indeed, in many GGCs, field stars, and cluster members use to populate different 


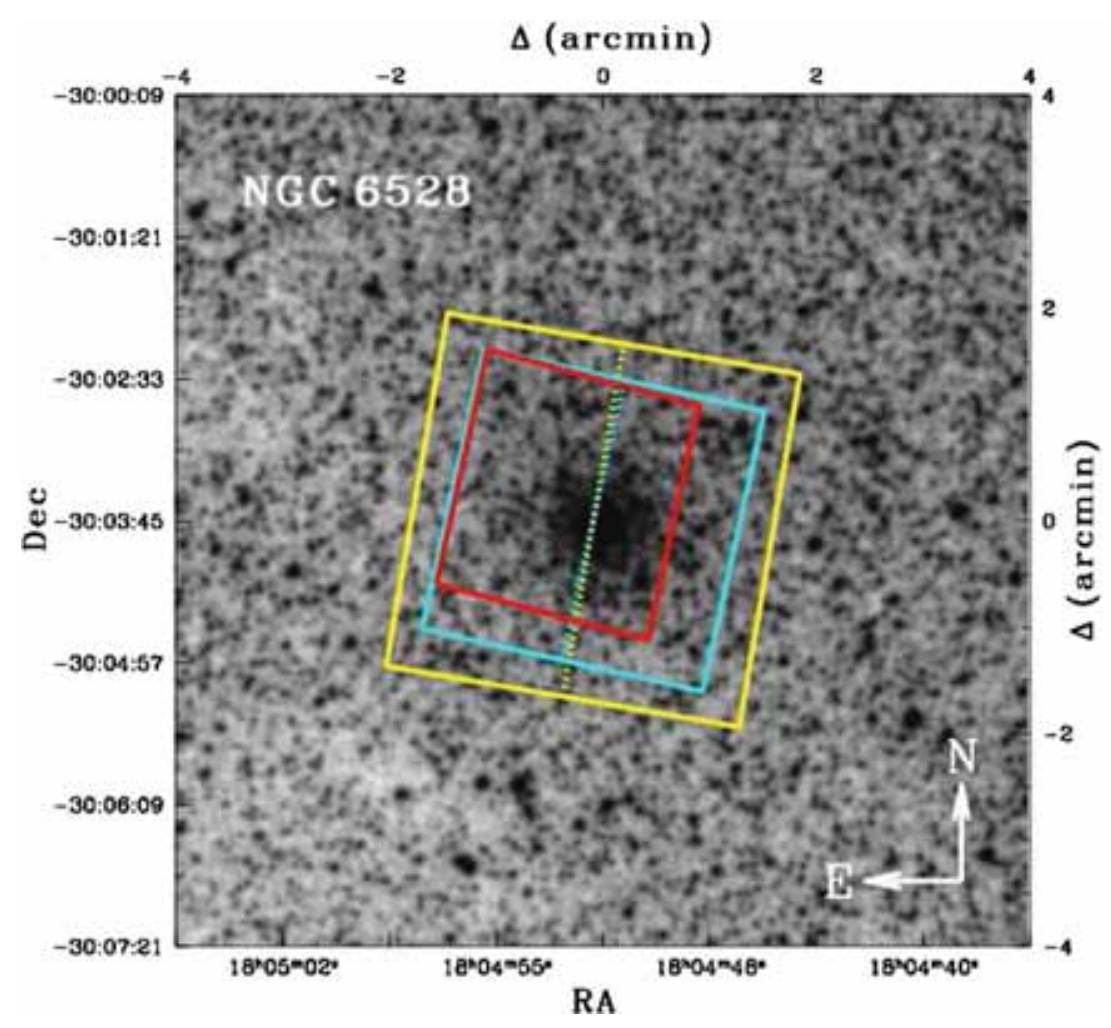

Figure 1. Footprints of optical and NIR HST images superimposed on a $8^{\prime} \times 8^{\prime}$ DSS red POSS-II plate. The yellow, cyan, and red boxes display the ACS/WFC, the WFC3/UVIS, and the WFC3/IR pointings. The orientation is north up and east left.

(A color version of this figure is available in the online journal.)

regions of the optical-NIR color-color plane. Unfortunately, the metallicity distribution of NGC 6528, bulge and thin-disk stars all peak around solar chemical composition, thus making NGC 6528 almost indistinguishable from the field in many color-color plane.

An alternative approach to separate field and cluster members is based on the analysis of stellar proper motions (PMs). A partial separation of NGC 6528 field and cluster stars was performed for the first time by Feltzing \& Johnson (2002) by using accurate astrometric measurements from multi-epoch images taken with the Wide-Field Planetary Camera 2 on board the Hubble Space Telescope (HST). A similar approach was also adopted by Zoccali et al. (2001) to constrain the bulge membership of NGC 6553.

In this investigation, we exploit the unique astrometric and photometric performance of the Ultraviolet and Visual Channel of the Wide Field Camera 3 (UVIS/WFC3), the Infrared Channel of WFC3 (IR/WFC3) and of the Advanced Camera for Survey (ACS) of HST to obtain high-accuracy astrometry and photometry of stars in the NGC 6528 FOV. PMs derived from this data set allowed us to separate, with unprecedented precision, most of the field stars from cluster members. The field-stars decontamination of the color-magnitude diagram (CMD) allowed us to correct the photometry of NGC 6528 for differential reddening, hence to derive an accurate age estimate by comparing the CMD of NGC 6528 with isochrones.

The structure of the paper is as follows. In Section 2 we present both the optical and the NIR data sets used in the current investigation together with the approach adopted to perform the photometry. The approach adopted to measure the PM and to select candidate cluster stars are discussed in Section 2.1, while Section 2.2 deals with the method adopted to estimate the differential reddening.

The selection of the candidate field stars is presented in Section 3, while in Section 4 we discuss the optical CMDs of candidate field and cluster stars together with advanced evolutionary features (red HB, RGB bump). In Section 5, we discuss in detail the comparison with two empirical calibrators: the old, metal-rich globular 47 Tuc and the super-metal-rich open cluster NGC 6791. Section 6 deals with the comparison with cluster isochrones and the difference with the age and the metallicity of the empirical calibrators. A brief summary of the results and a few possible avenues of the current project are outlined in Section 7.

\section{OBSERVATIONS AND DATA REDUCTION}

The data presented in this investigation come from HST archive images centered on NGC 6528. The ACS/WFC data set was collected on 2002 June $6,{ }^{14}$ in the filters $F 606 \mathrm{~W}(1 \times$ $4 \mathrm{~s}, 1 \times 50 \mathrm{~s}, 1 \times 450 \mathrm{~s})$, and $F 814 W(1 \times 4 \mathrm{~s}, 1 \times 50 \mathrm{~s}, 1 \times 450 \mathrm{~s})$. The WFC3 data set was collected between 2010 June 26 and 27, and includes optical and NIR images. The former were taken with the Ultraviolet and Visual channel (WFC3/UVIS $)^{15}$ in the filters $F 390 \mathrm{~W}(2 \times 40 \mathrm{~s}, 2 \times 348 \mathrm{~s}, 2 \times 715 \mathrm{~s}), F 555 \mathrm{~W}$ $(1 \times 1 \mathrm{~s}, 1 \times 50 \mathrm{~s}, 1 \times 665 \mathrm{~s})$, and $F 814 W(1 \times 1 \mathrm{~s}, 1 \times 50 \mathrm{~s}, 2 \times$ 370 s). The latter were taken with the WFC3 - Infrared channel (WFC3/IR) in the filters $F 110 W(3 \times 49 \mathrm{~s}, 1 \times 299 \mathrm{~s}, 2 \times 399 \mathrm{~s})$, and $F 160 W(3 \times 49 \mathrm{~s}, 1 \times 299 \mathrm{~s}, 2 \times 399 \mathrm{~s})$. The footprints of the different data sets are shown in Figure 1.

\footnotetext{
14 Program GO 9453, PI: T. Brown.

15 Program GO 11664, PI: T. Brown.
} 


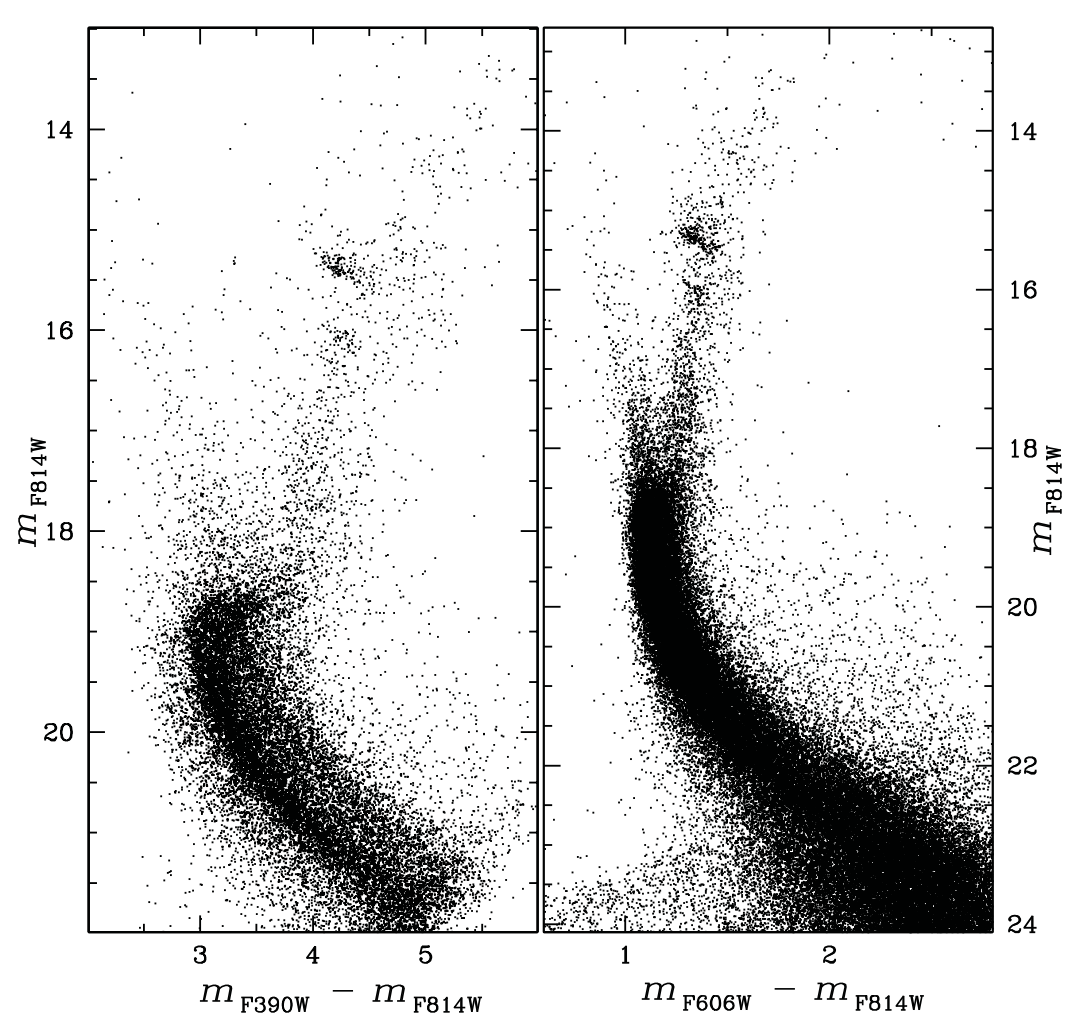

Figure 2. Optical CMD of stars in the field of view of NGC 6528 based either on WFC3/UVIS (left panel) or on ACS/WFC photometry (right panel).

In addition, to compare the CMD of NGC 6528 with that of the open cluster NGC 6791 we used ACS/WFC data in $F 606 \mathrm{~W}$ $(1 \times 0.5 \mathrm{~s}, 1 \times 5 \mathrm{~s}, 1 \times 50 \mathrm{~s})$, and $F 814 W(1 \times 0.5 \mathrm{~s}, 1 \times 5 \mathrm{~s}, 1 \times$ $50 \mathrm{~s})$ for NGC $6791 .{ }^{16}$

The photometric and astrometric reduction of ACS data, extensively described in Anderson et al. (2008), makes use of all the exposures simultaneously to generate a single list of stars, which are measured independently in each image. The routines used in the reduction exploit the independent dithered pointings of each image and the knowledge of the point spread function (PSF) to detect a star and avoid artifacts in the final star list. The reduction of the WFC3 images was performed with a software (img2xym_wfc3) that is mostly based on img2xym_WFC (Anderson \& King 2006). Star fluxes and positions were corrected for pixel area and geometric distortions using the solution given by Bellini et al. (2011) and finally calibrated into the VEGAmag as in Bedin et al. (2005).

Last, we selected a high-quality sample of stars, i.e., relatively isolated with small photometric, astrometric and PSF-fitting errors. For this selection we used the quality indices that our photometry software produces, in a procedure that is described in detail by Milone et al. (2009). By adopting the above selection criteria, the $\sim 20 \%$ of candidate cluster stars was rejected. The sample of objects that was neglected from the analysis includes: (1) stars that are poorly measured due to contamination by cosmic rays or bad pixels; (2) stars that are poorly measured due to contamination by nearby neighbor stars. Moreover, we also neglected non-stellar objects (background galaxies), hot pixels and artifacts associated to diffraction spikes of bright stars (see Anderson et al. 2008; Milone et al. 2009, 2012 for details). For these objects, we are not able to properly estimate photometric and astrometric (random

16 Program GO 10265, PI: T. Brown. and systematic) errors, because their profiles can strongly differ from the adopted PSF model.

We ended up with an accurate photometric and astrometric catalog of $\sim 210,000$ stars in a magnitude interval ranging from nearly the tip of the RGB to several magnitudes fainter than the main sequence turn-off (MSTO), reaching $m_{\mathrm{F} 814 \mathrm{~W}} \approx 24$. The $m_{\mathrm{F} 814 \mathrm{~W}}$ versus $m_{\mathrm{F} 390 \mathrm{~W}}-m_{\mathrm{F} 814 \mathrm{~W}} \mathrm{CMD}$ from WFC3/UVIS data and the $m_{\mathrm{F} 814 \mathrm{~W}}$ versus $m_{\mathrm{F} 606 \mathrm{~W}}-m_{\mathrm{F} 814 \mathrm{~W}} \mathrm{CMD}$ from ACS/WFC data are shown in Figure 2 for stars in the NGC 6528 FOV.

\subsection{Proper Motions}

PMs are obtained by comparing the position of stars measured at two different epochs, following an approach that has already been used in several papers (e.g., McLaughlin et al. 2006; Anderson \& King 2003; Bedin et al. 2001). In the present analysis, the average positions of the stars obtained by the optical ACS data and by both optical and NIR WFC3 data, represent, respectively, the positions at the first and second epoch.

The computation of the geometric linear transformation between the coordinate system of the first- and second-epoch catalogs is crucial for the determination of the PM of the stars in our FOV. Therefore, we started off with the identification of the same stars detected in both the epochs. Then, we picked up a subsample made up only by the ones unsaturated and with a high signal-to-noise ratio in all the ACS and WFC3 images. Moreover, since the cluster internal motions are negligible compared to the field star motions, we eventually decided to use cluster members as reference stars to define the transformation we are looking for.

In order to select the sample of cluster members as reference stars, we started by identifying all the stars that, on the basis of their position in the CMD, are probable cluster members, and obtained a raw PM estimate by using a local transformation based on this sample. As an example of the criteria of selection 

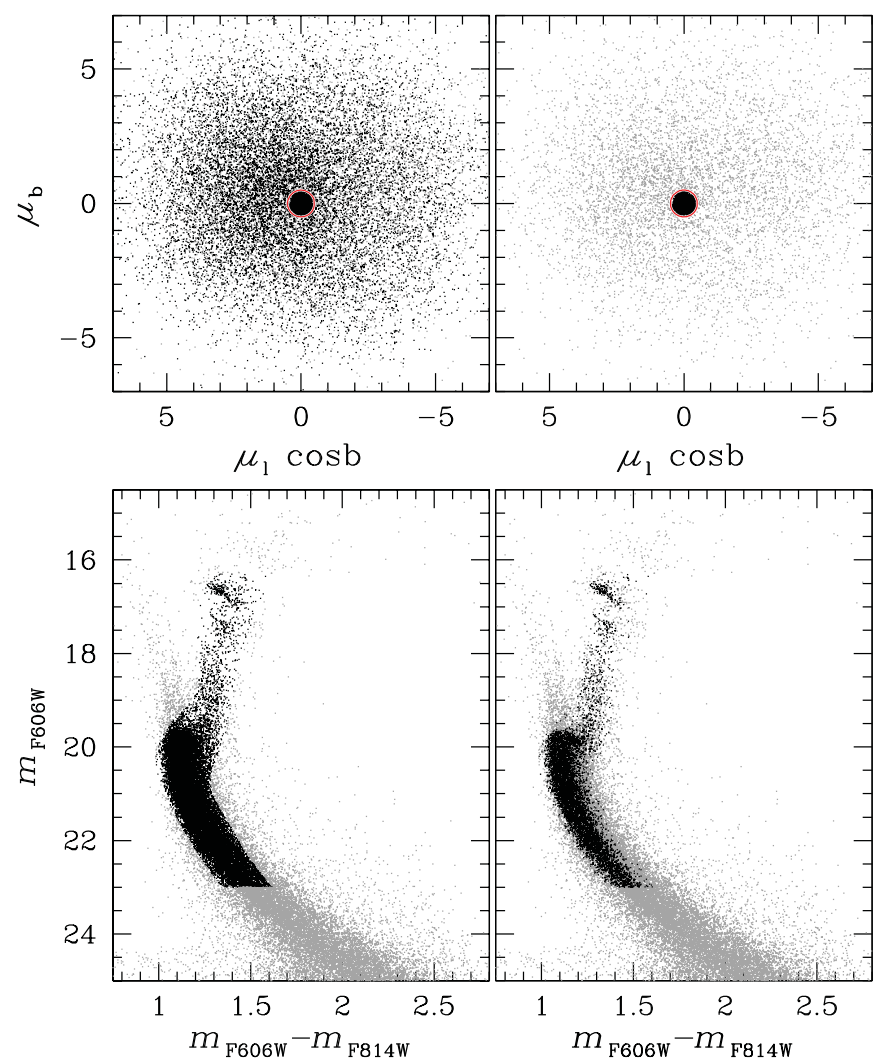

Figure 3. Proper motion of stars in the field of view of NGC 6528. The stars used as reference to measure proper motions are marked as black points in the CMDs (bottom panels) and in the vector-point diagram (top panels). The left panels show the sample of reference stars selected only on the basis of their position in the CMD and used to obtain a first preliminary estimate of proper motions. The right panels display the sample of reference stars used for the final measurement of proper motions and selected on the basis of their position both in the CMD and in the vector-point diagram.

(A color version of this figure is available in the online journal.)

we used, in the bottom left panel of Figure 3, we plotted with black points all the stars that are used as reference to obtain the first PM estimate. The same stars are plotted with the same color code in the vector-point diagram (VPD) shown in the topleft panel. It is clear that the PM of some reference stars is not consistent with their cluster membership.

At this point, we drew the red circle which has a radius $3 \sigma$, where $\sigma$ is the average PM dispersion for reference stars in each direction and contains the bulk of cluster stars. We excluded from the sample of reference stars all the stars that lie outside the red circle and do not have cluster-like PM even if they are placed near the cluster sequence. This ensures that the relative motion of the cluster stars is zero within the measurement errors.

Results are shown in Figure 4. In the bottom left panel, we plotted the VPD of the PMs in Galactic coordinates for stars located across the ACS FOV of NGC 6528: since PMs were measured relative to a sample of cluster members, the zero point of the motion is the mean motion of the cluster. A zoomed VPD around the origin is shown in the inset. The bulk of stars clustered around $\left(\mu_{1} \cos b, \mu_{\mathrm{b}}\right)=(0,0)$ mainly includes candidate cluster members while field stars are distributed over a broad range of PMs.

In the top right panel of Figure 4, a smoothed version of the VPD is plotted, and the contours are superimposed onto the diagram. The PM distribution of field stars is far from being symmetric across the four quadrants. The left quadrants (II and
III) show clear overdensities when compared with the right quadrants (I and IV) and the density of the field stars is maximum in the second quadrant as indicated by the contours. This evidence is also supported by the marginals of the distribution drawn in the top left $\left(\mu_{1} \cos b\right)$ and in the bottom right $\left(\mu_{\mathrm{b}}\right)$ panel. The above distributions are skewed, and indeed, the slopes at positive Galactic latitudes/longitudes are steeper than at negative ones. The mode of the field-star VPD also seems to be displaced toward positive latitudes and longitudes, relative to the cluster mode. A further analysis of the VPD is provided in Section 3.

\subsection{Differential Reddening}

The mean reddening in the direction of NGC 6528 is $E(B-V) \sim 0.54 \mathrm{mag}$ (Harris 1996, as updated in 2010), and it is not uniform across the FOV. Figure 2 indeed shows that all the evolutionary sequences of NGC 6528 are broadened. The main culprit ought to be the differential reddening.

To minimize this effect on our analysis, we corrected our photometry for differential reddening by using the method by Milone et al. (2012, see their Section 3.1). We note that the strong contamination from bulge and disk stars that are present in the FOV of NGC 6528 can significantly affect results of this procedure. To minimize the effect of field-stars contamination, we determined the amount of differential reddening by using a sample of stars that mainly including cluster members.

Candidate cluster members have been separated from field stars by following the approach outlined in Figure 5. The small boxes located in the right panel display the VPD of the stars in the range $13 \lesssim m_{\mathrm{F} 814 \mathrm{~W}} \lesssim 22$, divided into six bins of about 1.5 mag each (as indicated by horizontal dotted lines in the left panel). For the sake of clarity, we zoomed the VPD around the origin.

To identify the candidate cluster stars, we firstly selected in each box of Figure 5 a sample of stars with PM $\mu_{\mathrm{r}}=$

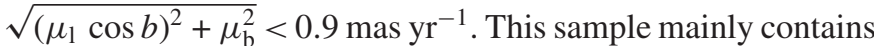
cluster members, but a mild contamination of field stars is still present.

To minimize the field-star contamination we evaluated the dispersion of PM distribution in each panel $(\sigma(i)$ with $i=1-6)$. Gray circles have radius $r(i)=3 \times \sigma(i)$, and we assumed cluster-members candidates the stars located inside the individual circles. The left panel of Figure 5 shows the $m_{\mathrm{F} 814 \mathrm{~W}}, m_{\mathrm{F} 390 \mathrm{~W}}-m_{\mathrm{F} 814 \mathrm{~W}} \mathrm{CMD}$ of the candidate cluster members.

The first step in the method used to correct for differential reddening is the evaluation of the main-sequence fiducial line in the CMD. Then we selected, for each star, the 45 nearest well-measured neighbors, in the catalog of candidate cluster stars, and evaluated for each of them the color distance from the fiducial line along the reddening line. We applied to the target star a correction equal to the median color distance of these 45 stars (details of the procedure are given in Milone et al. 2012).

The spatial reddening variation in the FOV of NGC 6528 is shown in Figure 6. Similarly to what done in Milone et al. (2012), we have divided the FOV into eight horizontal slices and eight vertical slices and plot $\Delta E(B-V)$ as a function of the $Y$ (top panels) and $X$ coordinate (right panels). We have also divided the whole FOV into $64 \times 64$ boxes of $128 \times 128 \mathrm{ACS} / \mathrm{WFC}$ pixels and calculated the average $\Delta E(B-V)$ within each of them. The resulting reddening map is displayed in the bottom left panel where each box is represented as a gray square. The levels 


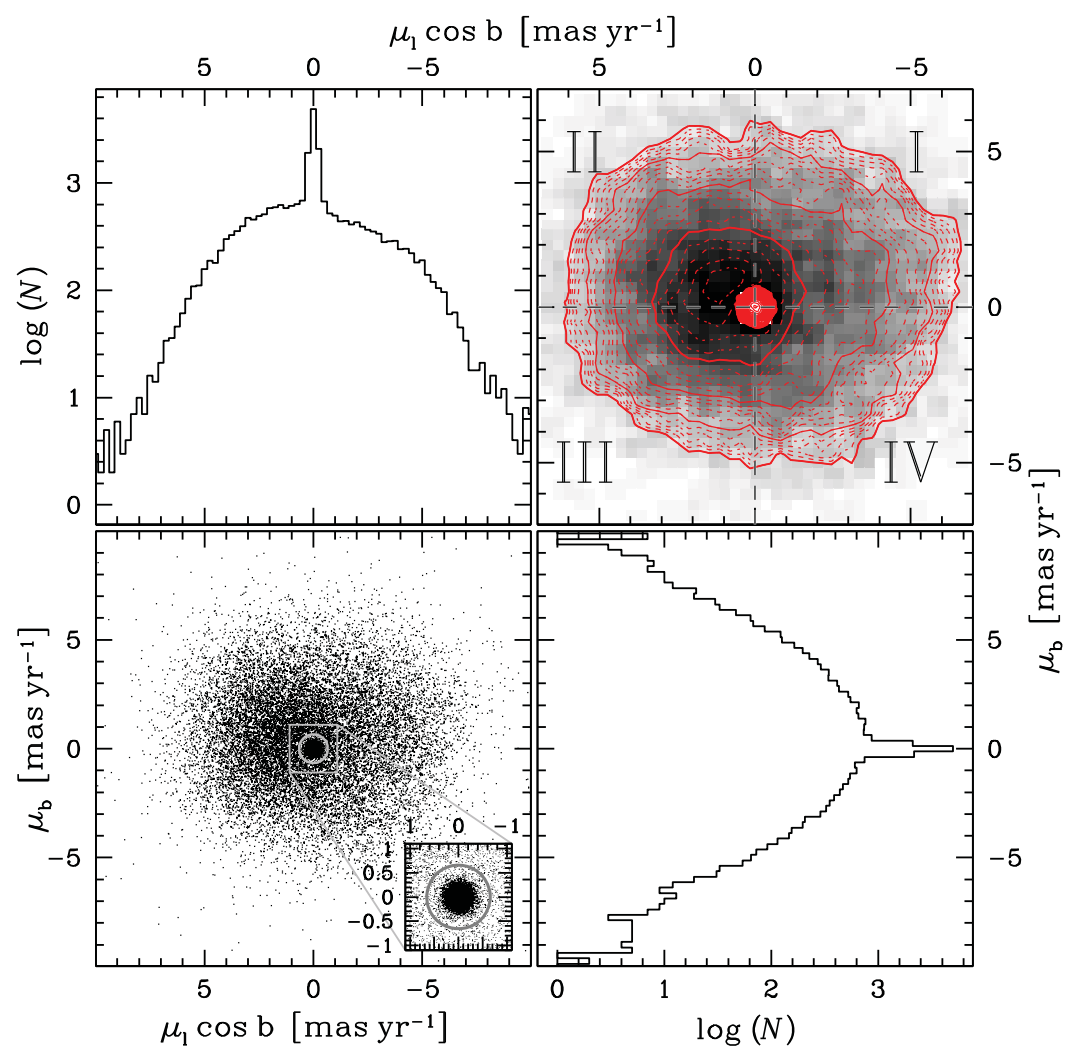

Figure 4. Bottom left: VPD of the proper-motion distribution of the entire sample of stars: for the sake of clarity only stars with $m_{\mathrm{F} 814 \mathrm{~W}}<22.1$ have been plotted across the FOV of NGC 6528. The inset shows a zoom-in around the origin of the VPD. The bulk of stars around $\left(\mu_{1} \cos b, \mu_{\mathrm{b}}\right)=(0,0)$ mainly consists of cluster members. Top right: smoothed VPD diagram and contours of the previous sample. The dashed lines separate the four quadrants. The top left and the bottom right panels show the marginals of the proper motions along the $\mu_{1} \cos b$, and the $\mu_{\mathrm{b}}$ axis.

(A color version of this figure is available in the online journal.)
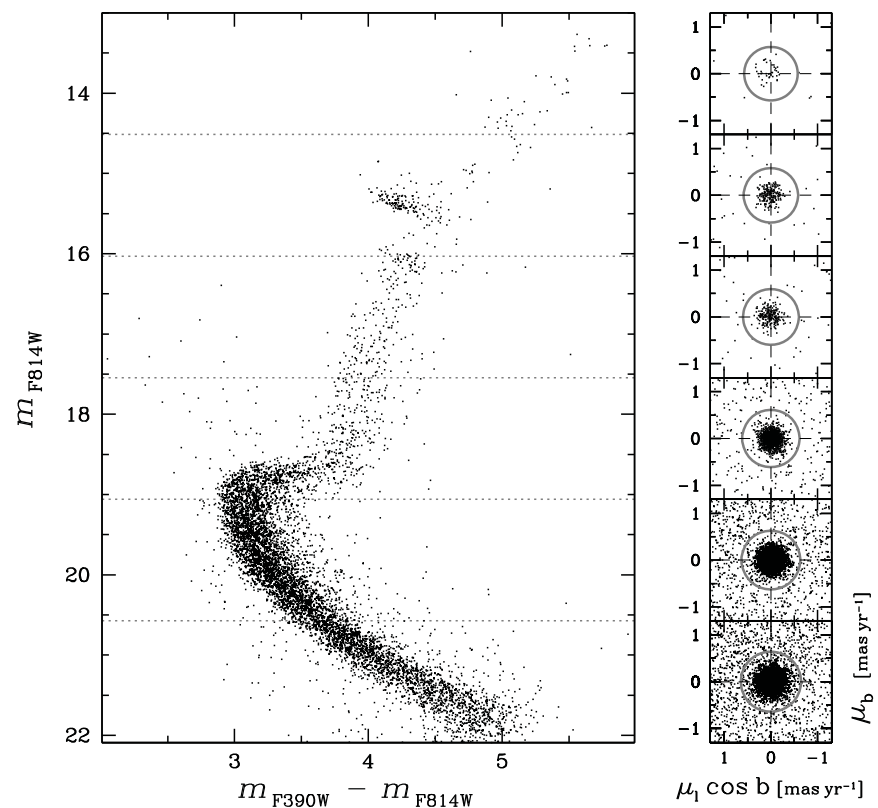

Figure 5. Left: $m_{\mathrm{F} 814 \mathrm{~W}}, m_{\mathrm{F} 390 \mathrm{~W}}-m_{\mathrm{F} 814 \mathrm{~W}} \mathrm{CMD}$ of candidate cluster stars. Right: VPD for the stars in six magnitude bins, marked by dotted lines in the left panel. Gray circle separate candidate cluster members from field stars (see the text for details).

of gray are indicative of the amount of differential reddening according to the scale on the top right. Figure 7 compares the original CMD for cluster-members candidates and the CMD corrected for differential reddening.

\section{SELECTION OF BULGE STARS}

In the previous section, we have described the procedure for the selection of the candidate cluster members. The $m_{\mathrm{F} 814 \mathrm{~W}}$, $m_{\mathrm{F} 390 \mathrm{~W}}-m_{\mathrm{F} 814 \mathrm{~W}} \mathrm{CMD}$ of the remaining field stars is plotted in Figure 8. A glance at this CMD reveals a blue MS above the TO region that is populated by young likely disk stars, and a spread red $\mathrm{RGB}$, that could be associated to the bulge.

In order to select a sample of candidate bulge members, among the field stars, we started to estimate the average motion of a small set of stars that, on the basis of their position in the CMD, are probable bulge members. In particular, we picked up a subsample of the $\sim 200$ brightest and reddest RGB stars, corresponding to the black crosses lying within the window outlined by the dashed lines.

The VPD of the selected stars has then been plotted (right bottom panel) and the median and the standard deviation of the PM distributions along the two Galactic coordinates evaluated, obtaining: $\left\langle\mu_{1} \cos b\right\rangle=+1.09 \pm 0.2 \mathrm{mas} \mathrm{yr}^{-1},\left\langle\mu_{\mathrm{b}}\right\rangle=+0.74 \pm$ $0.2 \mathrm{mas} \mathrm{yr}^{-1} ; \sigma_{\mu_{1} \cos b}=2.57 \pm 0.2 \mathrm{mas} \mathrm{yr}^{-1}, \sigma_{\mu_{\mathrm{b}}}=2.18 \pm$ $0.16 \mathrm{mas} \mathrm{yr}^{-1}$. Noticeably, the motion of the bulge is centered on the quadrant II, thus suggesting that the overdensity of stars observed in the VPD of Figure 4 and discussed in Section 2.1 is mainly due to the bulk motion of bulge stars. Our estimates of the bulge velocity dispersion are consistent with those provided by Feltzing \& Johnson (2002), who found $\sigma_{\mu_{1} \cos b}=3.27 \pm 0.27$ mas yr $^{-1}, \sigma_{\mu_{\mathrm{b}}}=2.54 \pm 0.17 \mathrm{mas} \mathrm{yr}^{-1}$ and Kuijken \& Rich (2002), who found $\sigma_{\mu_{1} \cos b}=2.98 \pm 0.017$ and $\sigma_{\mu_{\mathrm{b}}}=2.54 \pm 0.014 \mathrm{mas} \mathrm{yr}^{-1}$ for stars in the Baade's Window. 


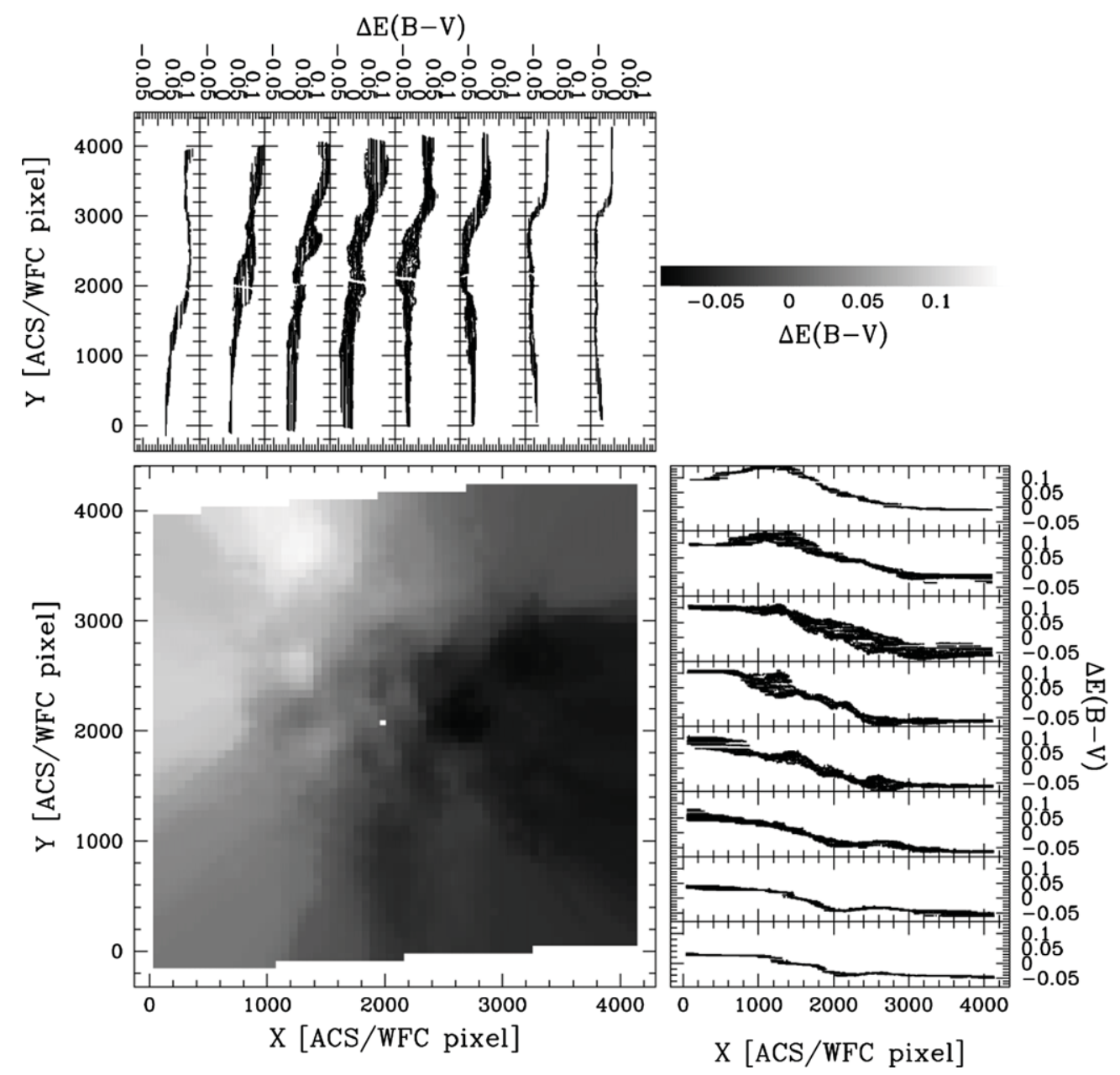

Figure 6. Bottom left: map of reddening across the FOV of NGC 6528. Gray levels indicate different values of reddening as indicated on the top right. The top and the bottom right panel display $\Delta E(B-V)$ as a function of $Y$ and $X$, respectively, for stars located in eight vertical and horizontal slices.

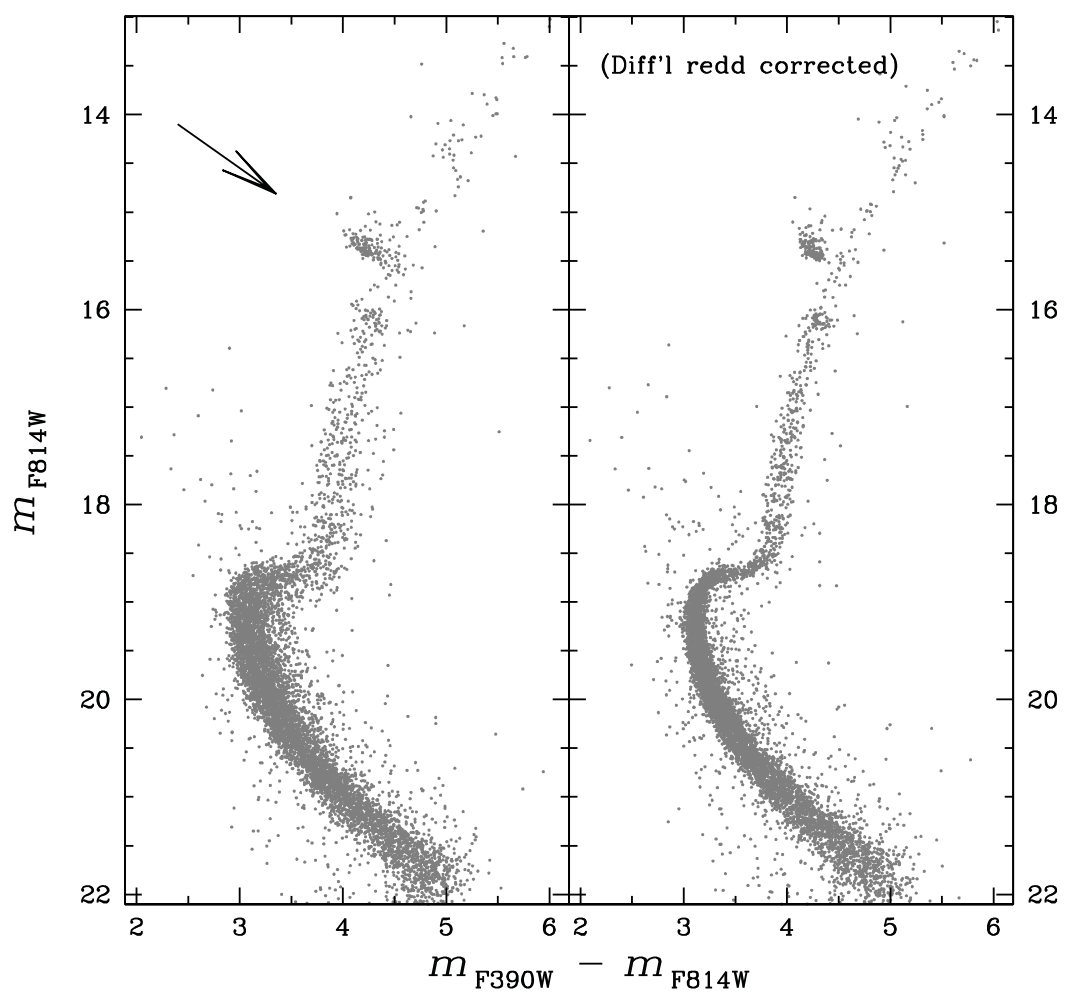

Figure 7. $m_{\mathrm{F} 814 \mathrm{~W}}, m_{\mathrm{F} 390 \mathrm{~W}}-m_{\mathrm{F} 814 \mathrm{~W}} \mathrm{CMD}$ of the candidate cluster members before (left) and after the reddening correction (right). 


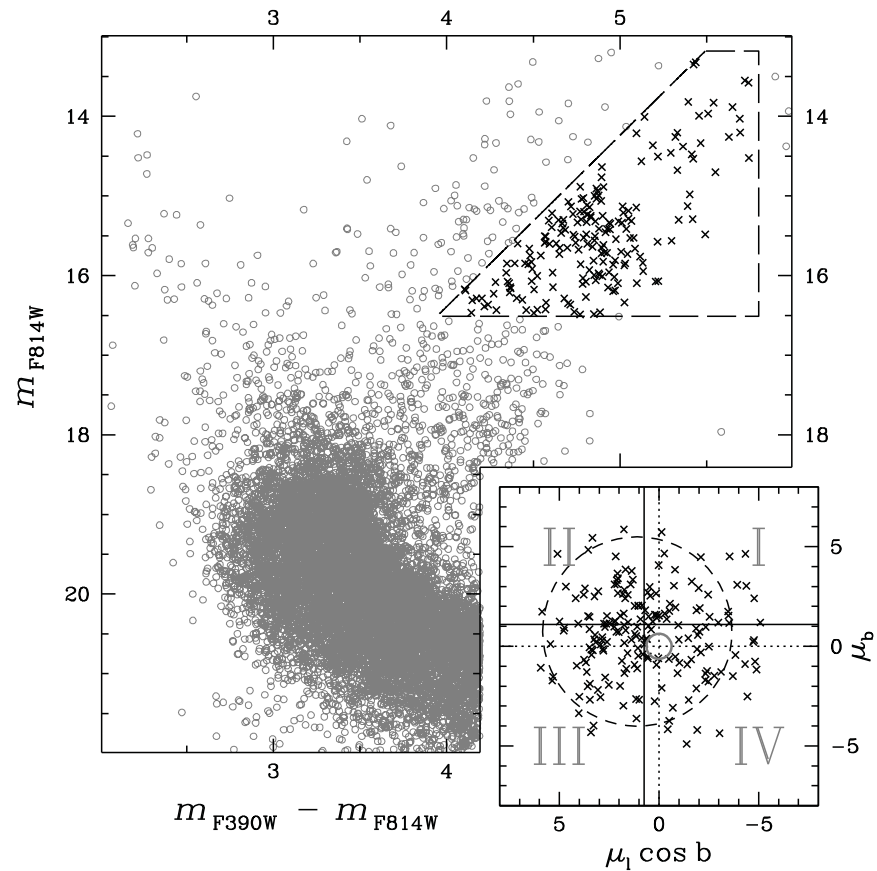

Figure 8. $m_{\mathrm{F} 814 \mathrm{~W}}, m_{\mathrm{F} 390 \mathrm{~W}}-m_{\mathrm{F} 814 \mathrm{~W}} \mathrm{CMD}$ of field stars. The black crosses located inside the dashed window, mark stars that, according to their position in the CMD, are probable bulge members. The position of these stars in the VPD is shown in the inset.

The bulge membership of the stars in our catalog has then been established following an approach similar to that used to select the candidates of cluster population, as displayed in the right panels of Figure 9. All the stars not belonging to NGC 6528, whose PMs are located inside a circle with a radius equal to $2 \times \sigma_{\mathrm{b}}$ where $\sigma_{\mathrm{b}}=2.38$ is the average value of the standard deviation along the two Galactic coordinates, are flagged as possible bulge members. The $m_{\mathrm{F} 814 \mathrm{~W}}$ versus $m_{\mathrm{F} 390 \mathrm{~W}}-m_{\mathrm{F} 814 \mathrm{~W}} \mathrm{CMD}$ of selected bulge stars is plotted in the left panel of Figure 9.

\section{THE CMDs OF NGC 6528 AND BULGE STARS}

In this section, we analyze the CMDs of candidate cluster and bulge stars. The left panel of Figure 10 shows the $m_{\mathrm{F} 814 \mathrm{~W}}$, $m_{\mathrm{F} 390 \mathrm{~W}}-m_{\mathrm{F} 814 \mathrm{~W}} \mathrm{CMD}$ of NGC 6528 . The selection of candidate cluster members based on PMs and, the correction for differential reddening improved the photometric precision and allowed us to disclose several interesting evolutionary features of NGC 6528.

1. The HB morphology is typical of metal-rich GGCs, and indeed central He-burning stars are located in a well defined red HB (red ellipse; $\left\langle m_{\mathrm{F} 814 \mathrm{~W}}\right\rangle=15.33,\left\langle m_{\mathrm{F} 390 \mathrm{~W}}-\right.$ $\left.\left.m_{\mathrm{F} 814 \mathrm{~W}}\right\rangle=4.25 \mathrm{mag}\right)$.

2. Stars located across the RGB bump (blue ellipse; $\left.\left\langle m_{\mathrm{F} 814 \mathrm{~W}}=16.10\right\rangle,\left\langle m_{\mathrm{F} 390 \mathrm{~W}}-m_{\mathrm{F} 814 \mathrm{~W}}\right\rangle=4.29 \mathrm{mag}\right)$ display a well defined overdensity. The luminosity - as expected in metal-rich GGCs-is fainter than the red HB.

3. Evolved (SGB, RGB) and unevolved (MS) evolutionary features display a tight distribution in color. Indeed, the spread in color around the cluster ridge line (red solid line) is typically smaller than $0.04 \mathrm{mag}$.

The CMD of candidate bulge is plotted in the right panel of Figure 10. The comparison with the cluster ridge line indicates that the bulk of bulge red giants (RGs) are systematically redder than cluster RGs, thus suggesting that they are-on
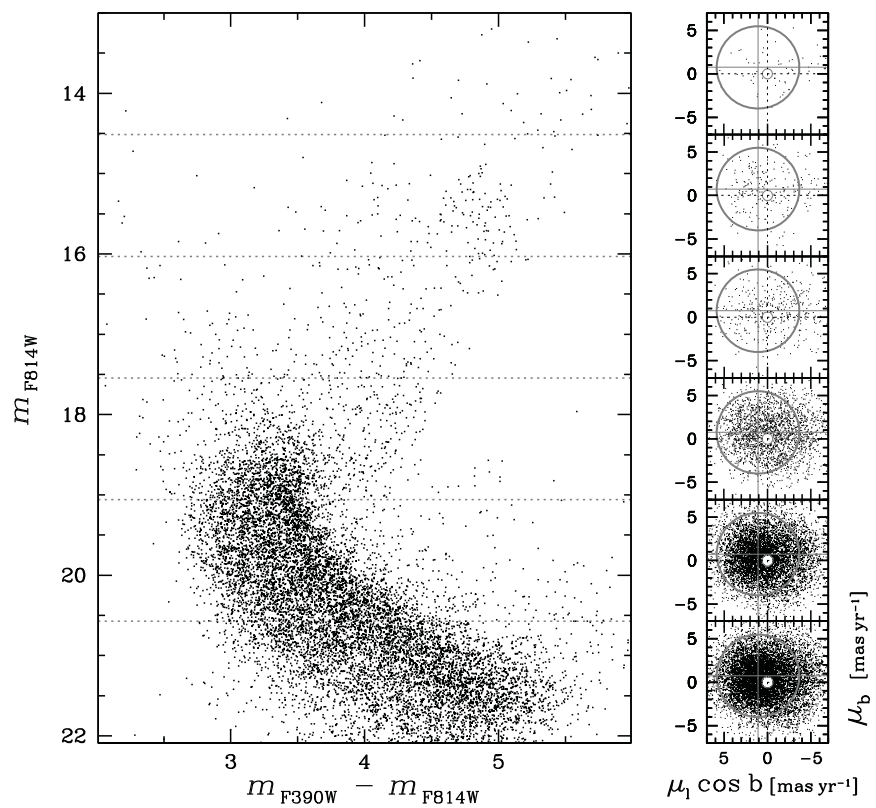

Figure 9. Left panel: $m_{\mathrm{F} 814 \mathrm{~W}}$ vs. $m_{\mathrm{F} 390 \mathrm{~W}}-m_{\mathrm{F} 814 \mathrm{~W}} \mathrm{CMD}$ for candidate bulge stars. Right panels: VPD of field stars in six magnitude bins. Gray circles separate candidate bulge stars from remaining field stars. See the text for details.

average-more metal-rich. This working hypothesis is also supported by the fact that the SGB of bulge stars $\left(m_{\mathrm{F} 814 \mathrm{~W}} \simeq\right.$ $18.75,3.6 \lesssim m_{\mathrm{F} 390 \mathrm{~W}}-m_{\mathrm{F} 814 \mathrm{~W}} \lesssim 4$ ) shows a flatter color distribution than cluster SGB stars. Moreover, bulge RGs show, at fixed luminosity, a larger spread in color when compared with cluster RGs. These empirical evidence suggests that bulge RGs are affected either by a more complex reddening distribution along the line of sight and/or by a larger spread in metallicity.

The MS of bulge stars is affected by a large spread in color, but the morphology of the MS across the TO region is quite similar to the cluster ridge line. The same outcome applies to red HB stars, and indeed they cover a broader range both in magnitude $\left(m_{\mathrm{F} 814 \mathrm{~W}} \approx 15.2 \div 16.2\right)$ and in color $\left(m_{\mathrm{F} 390 \mathrm{~W}}-m_{\mathrm{F} 814 \mathrm{~W}} \approx 4.7 \div 5.1\right)$. Moreover, the blue MS $\left(m_{\mathrm{F} 814 \mathrm{~W}} \leqslant 18.5, m_{\mathrm{F} 390 \mathrm{~W}}-m_{\mathrm{F} 814 \mathrm{~W}} \leqslant 3.40\right)$ tracing a young disk population seems poorly populated. The above evidence confirms that residual contamination from the disk is negligible for our purposes and the field stars we are dealing with are mainly bulge stars. Moreover, the latter ones appear to be coeval with candidate cluster stars, thus supporting earlier findings by Ortolani et al. (2001).

More quantitative estimates of the bulge stellar population(s) are however hampered by the notorious degeneracies among differential reddening, metallicity and stellar ages that are typical of optical CMDs.

\section{EMPIRICAL CONSTRAINTS}

To constrain the evolutionary properties of cluster and field stars, we adopted two empirical calibrators. We selected the old, metal-rich GGC 47 Tucanæ $=\mathrm{NGC} 104(t \approx 11 \mathrm{Gyr},[\mathrm{Fe} / \mathrm{H}]=$ -0.8; VandenBerg et al. 2010; Carretta et al. 2009) and the old, super-metal-rich open cluster NGC $6791(t \approx 8 \mathrm{Gyr},[\mathrm{Fe} / \mathrm{H}]=$ +0.3; Brasseur et al. 2010; Boesgaard et al. 2009). For 47 Tuc we used accurate ACS/WFC photometry in $F 606 \mathrm{~W}$ and $F 814 \mathrm{~W}$ bands from Anderson et al. (2008) and Calamida et al. (2012). 


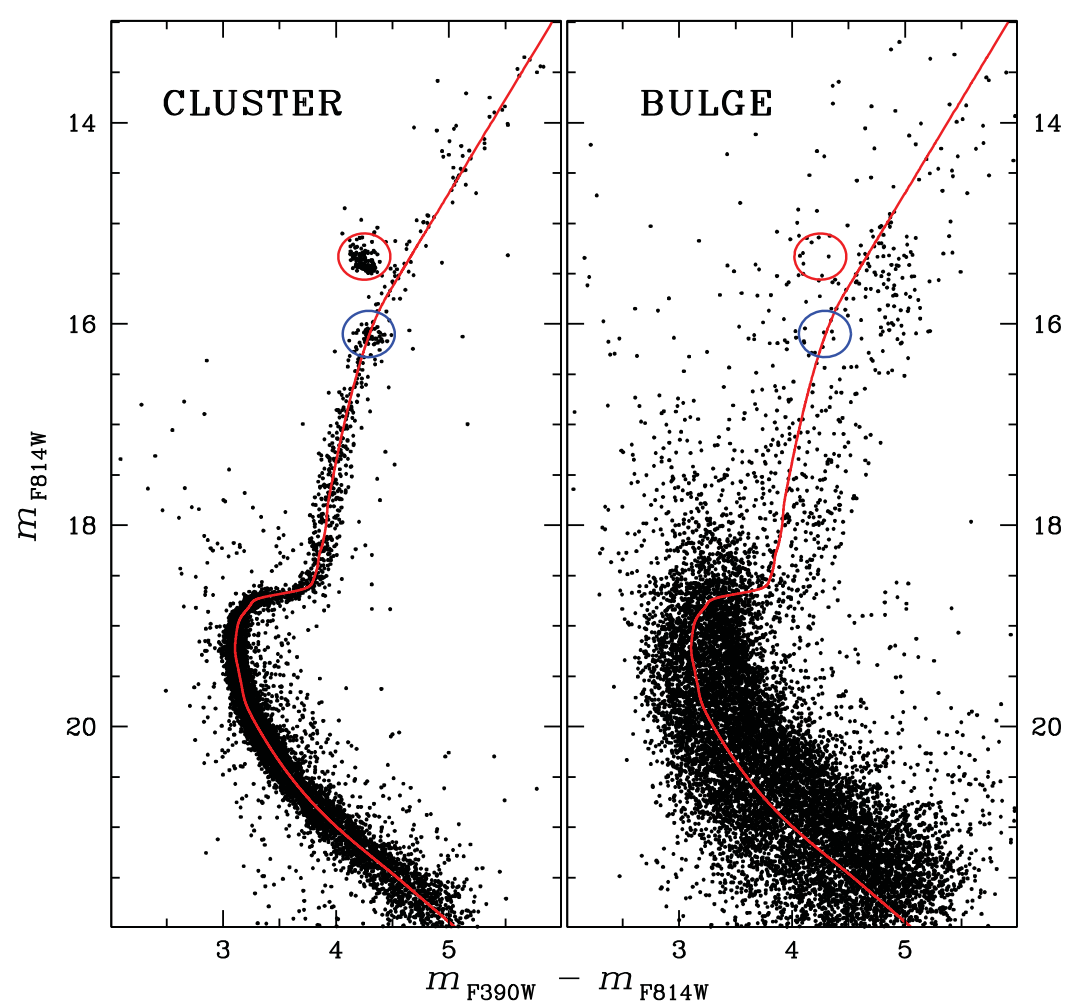

Figure 10. Left panel: $m_{\mathrm{F} 814 \mathrm{~W}}$ vs. $m_{\mathrm{F} 390 \mathrm{~W}}-m_{\mathrm{F} 814 \mathrm{~W}} \mathrm{CMD}$ of candidate cluster (NGC 6528) members. The red solid line shows the ridge line of NGC 6528, while the red and the blue ellipses mark the position of red HB stars and of RGB bump stars. Right panel: same as the left, but for bulge stars. The line and the symbols are the same of the left panel.

(A color version of this figure is available in the online journal.)

The photometry of NGC 6791, has been performed for this investigation (see Section 2). ${ }^{17}$

Data plotted in the top panels of Figure 11 display the $m_{\mathrm{F} 814 \mathrm{~W}}, m_{\mathrm{F} 606 \mathrm{~W}}-m_{\mathrm{F} 814 \mathrm{~W}} \mathrm{CMD}$ of 47 Tuc (left) and of NGC 6791 (right). The red and blue circles in the left panel identify, respectively, the red $\mathrm{HB}\left(\left\langle m_{\mathrm{F} 814 \mathrm{~W}}\right\rangle=13.00,\left\langle m_{\mathrm{F} 606 \mathrm{~W}}-\right.\right.$ $\left.\left.m_{\mathrm{F} 814 \mathrm{~W}}\right\rangle=0.68 \mathrm{mag}\right)$ and the RGB bump $\left(\left\langle m_{\mathrm{F} 814 \mathrm{~W}}\right\rangle=\right.$ $\left.13.5,\left\langle m_{\mathrm{F} 606 \mathrm{~W}}-m_{\mathrm{F} 814 \mathrm{~W}}\right\rangle=0.77 \mathrm{mag}\right)$, while the green curve shows the ridge line of the cluster. Symbols in the right panel are the same as in the left panel, but the red circle centered on $m_{\mathrm{F} 814 \mathrm{~W}}=13.2, m_{\mathrm{F} 606 \mathrm{~W}}-m_{\mathrm{F} 814 \mathrm{~W}}=1.03$ mag marks red clump stars (the ridge line is shown in magenta). The RGB bump was not identified, since the number of stars along the RGB in this cluster is quite limited.

To compare the different clusters we adopted for NGC 6528 a true distance modulus of 14.50 and a cluster reddening of $E(B-V)=0.56 \mathrm{mag}$ (Harris 1996). These values are consistent with the estimates available in the literature $\left(\mu_{0}=15.10, E(B-\right.$ $V)=0.46$, Zoccali et al. 2004; $\mu_{0}=14.49, E(B-V)=0.54$, Momany et al. 2003). The reddening was transformed into the ACS bands by interpolating the extinction values provided in Bedin et al. (2005), and we found $E(F 606 W-F 814 W) \simeq$ $0.53 \mathrm{mag}$.

The comparison between candidate members of NGC 6528 and the fiducial line of 47 Tuc (middle left panel) seems to indicate that the former is more metal-rich than the latter. The

\footnotetext{
17 Note that the above clusters were selected because our group already performed accurate WFC3/IR photometry (Calamida et al. 2012). This means that we are following the very same approach to perform the photometry and to derive the cluster ridge lines. Moreover, we plan to use the same empirical calibrators for both optical and NIR photometry.
}

hypothesis that the difference is mainly in chemical composition and not in cluster age is supported by the following evidence.

1. The entire MS in 47 Tuc is systematically bluer than in NGC 6528.

2. The RGB in NGC 6528 has a shallower slope than the RGB of 47 Tuc.

3. Both the red HB and the RGB bump of 47 Tuc are brighter and bluer than those in NGC 6528.

On the other hand, the comparison between NGC 6528 and NGC 6791 indicates that the former cluster is less metal-rich than the latter. The hypothesis that the difference is in both chemical composition and age is supported by the following evidence.

1. The ridge line of NGC 6791 becomes, for magnitudes fainter than $m_{\mathrm{F} 814 \mathrm{~W}} \sim 21.5$, systematically redder than MS stars in NGC 6528. Moreover, the ridge line of NGC 6791 attains colors in the TO region that are systematically brighter than MSTO stars in NGC 6528.

2. The shape and the extent in color of the sub-giant branch region in NGC 6791 is narrower $\left(m_{\mathrm{F} 606 \mathrm{~W}}-m_{\mathrm{F} 814 \mathrm{~W}} \sim 0.85\right.$ vs. $\left.m_{F 606 W}-m_{\mathrm{F} 814 \mathrm{~W}} \sim 1.2\right)$ compared with NGC 6528 .

The comparison with the candidate bulge stars does not permit firm constraints on their evolutionary properties. However, field RGs are systematically redder and display a shallower slope when compared with the 47 Tuc ridge line. Moreover, the distribution in magnitude and in color of field SGB stars are narrower than in 47 Tuc. The above evidence indicates that bulge stars are also more metal-rich than 47 Tuc (bottom left panel of Figure 11). 

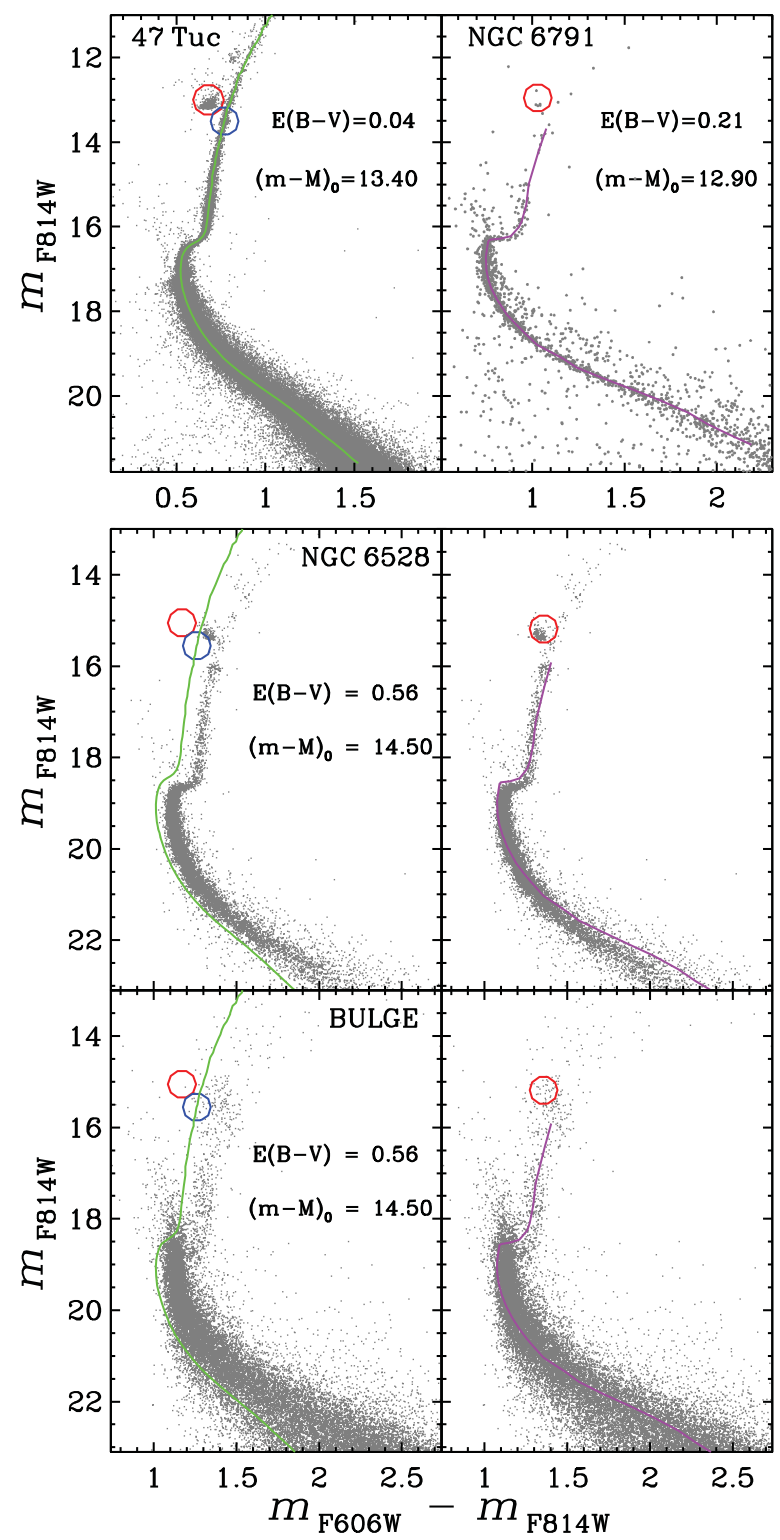

Figure 11. Top left: $m_{\mathrm{F} 814 \mathrm{~W}}, m_{\mathrm{F} 606 \mathrm{~W}}-m_{\mathrm{F} 814 \mathrm{~W}} \mathrm{CMD}$ of 47 Tuc based on images collected with ACS at HST. The red and blue circles mark, respectively, the red $\mathrm{HB}$ and the RGB bump, while the green line the cluster ridge line. Top right: same as the left, but for the old, metal-rich cluster NGC 6791. The red circle marks the position of red clump stars. Middle left: comparison between the $m_{\mathrm{F} 814 \mathrm{~W}}, m_{\mathrm{F} 606 \mathrm{~W}}-m_{\mathrm{F} 814 \mathrm{~W}} \mathrm{CMD}$ of NGC 6528 and the ridge line of 47 Tuc. The adopted true distance modulus and reddening (Harris 1996) are labeled. Middle right: same as the left, but the comparison is with the old, metal-rich cluster NGC 6791. Bottom left: same as the middle, but the comparison is between candidate bulge stars and the ridge line of 47 Tuc. Bottom right: same as the left, but the comparison is with the old, metal-rich cluster NGC 6791.

(A color version of this figure is available in the online journal.)

Data plotted in the bottom right panel of Figure 11 show a plausible agreement with the ridge line of NGC 6791, and indeed the slope of the cluster RGB is quite similar to bulge RG stars. Moreover, field MSTO stars attain colors $\left(m_{\mathrm{F} 606 \mathrm{~W}}-m_{\mathrm{F} 814 \mathrm{~W}} \sim\right.$ $1.1)$ and magnitudes $\left(m_{\mathrm{F} 814 \mathrm{~W}} \sim 18.5-19.5\right)$ similar to the ridge line of NGC 6791. The above evidence indicates that bulge stars might have a spread in age of the order of a few Gyr. Current findings rely on a very limited fraction of bulge stars, more quantitative constraints do require accurate and deep photometry and PM estimates along different line of sights of both the inner and the outer bulge.

\section{THEORETICAL CONSTRAINTS}

We computed stellar tracks and isochrones with metallicities $Z=0.004,0.010,0.015,0.020,0.025,0.035$. The initial helium abundance was fixed by assuming a linear helium-to-metal enrichment ratio: $Y=Y_{\mathrm{p}}+\Delta Y / \Delta Z Z$, with a cosmological He abundance $Y_{\mathrm{p}}=0.2485$ (Steigman 2006; Peimbert et al. 2007) and $\Delta Y / \Delta Z=2$ (Pagel \& Portinari 1998; Jimenez et al. 2003; Gennaro et al. 2010).

The different sets of evolutionary models were constructed assuming the following chemical compositions: $(Z, Y)$ : 0.004, $0.256 ; 0.010,0.269 ; 0.015,0.278 ; 0.020,0.288 ; 0.025,0.299$; $0.030,0.308$. We adopted both scaled-solar Asplund et al. (2009) and $\alpha$-enhanced $([\alpha / \mathrm{Fe}]=+0.3)$ chemical mixtures. For each set, the evolutionary models were computed for masses ranging from 0.30 to $1.20 M_{\odot}\left(\Delta M=0.05 M_{\odot}\right)$. Three different values of the mixing length parameter- $\alpha_{\mathrm{ml}}=1.7,1.8,1.9$-were adopted. This large grid of models was computed with the FRANEC evolutionary code (Pisa Stellar Evolution Data Base, Dell'Omodarme et al. 2012; Degl'Innocenti et al. 2008). The stellar isochrones range from 5 to 15 Gyr. To transform evolutionary prescriptions into the observational plane, we adopted the synthetic spectra by Brott \& Hauschildt (2005) for $T_{\text {eff }} \leqslant$ $10,000 \mathrm{~K}$ and by Castelli \& Kurucz (2003) for $T_{\text {eff }}>10,000 \mathrm{~K}$. The synthetic spectra were computed assuming a scaled-solar chemical mixture and have the same global metallicity of evolutionary models.

To validate the cluster isochrones, we estimated the age of the two calibrating clusters. Top panels of Figure 12 show the comparison in the $m_{\mathrm{F} 814 \mathrm{~W}}, m_{\mathrm{F} 606 \mathrm{~W}}-\mathrm{F} 814 \mathrm{~W}$ CMD between isochrones and observations for 47 Tuc (right) and NGC 6791 (left). The isochrones that provide the best fit with 47 Tuc data are based on evolutionary models constructed assuming a mixing length $\alpha_{\mathrm{ml}}=1.9$, an $\alpha$-enhanced $([\alpha / \mathrm{Fe}]=+0.30)$ chemical mixture and a metal-rich $([\mathrm{Fe} / \mathrm{H}]=-0.72)$ composition. We assumed for 47 Tuc the distance modulus and the reddening labeled in Figure 11. The comparison between isochrones and observations shown in Figure 12 suggest that the absolute age of 47 Tuc is $\sim 11 \pm 1 \mathrm{Gyr}$, in agreement with previous estimates from different authors. ${ }^{18}$

The isochrones to constrain the age of the open cluster NGC 6791 were constructed by assuming a scaledsolar $([\alpha / \mathrm{Fe}]=0)$ chemical mixture and a super-metal-rich $([\mathrm{Fe} / \mathrm{H}]=0.40)$ composition. We used the same distance modulus and reddening labeled in Figure 11 and infer that the absolute age of NGC 6791 is $\sim 8 \pm 1$ Gyr in agreement with similar literature estimates. ${ }^{19}$

To constrain the absolute age of NGC 6528, we performed a series of fits using different chemical mixtures and different iron abundances. The bottom-left panel of Figure 12 shows the comparison of the observed $F 814 W, F 606 W-F 814 W$ CMD with best-fit isochrones. Data plotted in this panel indicate

\footnotetext{
18 The literature concerning distance modulus, reddening and age estimates of 47 Tuc is quite broad: $(m-M)_{0}=13.18 \pm 0.07, E(B-V)=0.04 \pm 0.02$, $t \approx 11.5 \mathrm{Gyr}$, (Salaris et al. 2007); $(m-M)_{\mathrm{V}} \simeq 13.375, E(B-V) \simeq 0.04$ $t \approx 12$ Gyr, (Bergbusch \& Stetson 2009); $(m-M)_{\mathrm{V}} \simeq 13.40$, $E(B-V) \simeq 0.032, t \approx 11 \mathrm{Gyr}$, (VandenBerg et al. 2010); $(m-M)_{\mathrm{V}}=13.30, E(B-V)=0.03, t=12.75 \pm 0.5$ Gyr $($ Dotter et al. 2010), $t=11.75 \pm 0.25$ Gyr (VandenBerg et al. 2013); and with Marín-Franch et al. (2009): 12.3-13.7 Gyr, using different sets of isochrones. More recently, Hansen et al. (2013) have inferred a younger age of $t=9.9 \pm 0.7 \mathrm{Gyr}$ from their analysis of the white dwarf cooling sequence.

19 The literature distance modulus, reddening and age estimates of NGC 6791 cover a broad range: $(m-M)_{0} \sim 13.57, E(B-V) \sim 0.15, t \approx 8 \mathrm{Gyr}$, (Brasseur et al. 2010); $(m-M)_{\mathrm{V}} \sim 13.51, E(B-V) \sim 0.14, t \approx 8.3 \mathrm{Gyr}$, (Brogaard et al. 2012).
} 

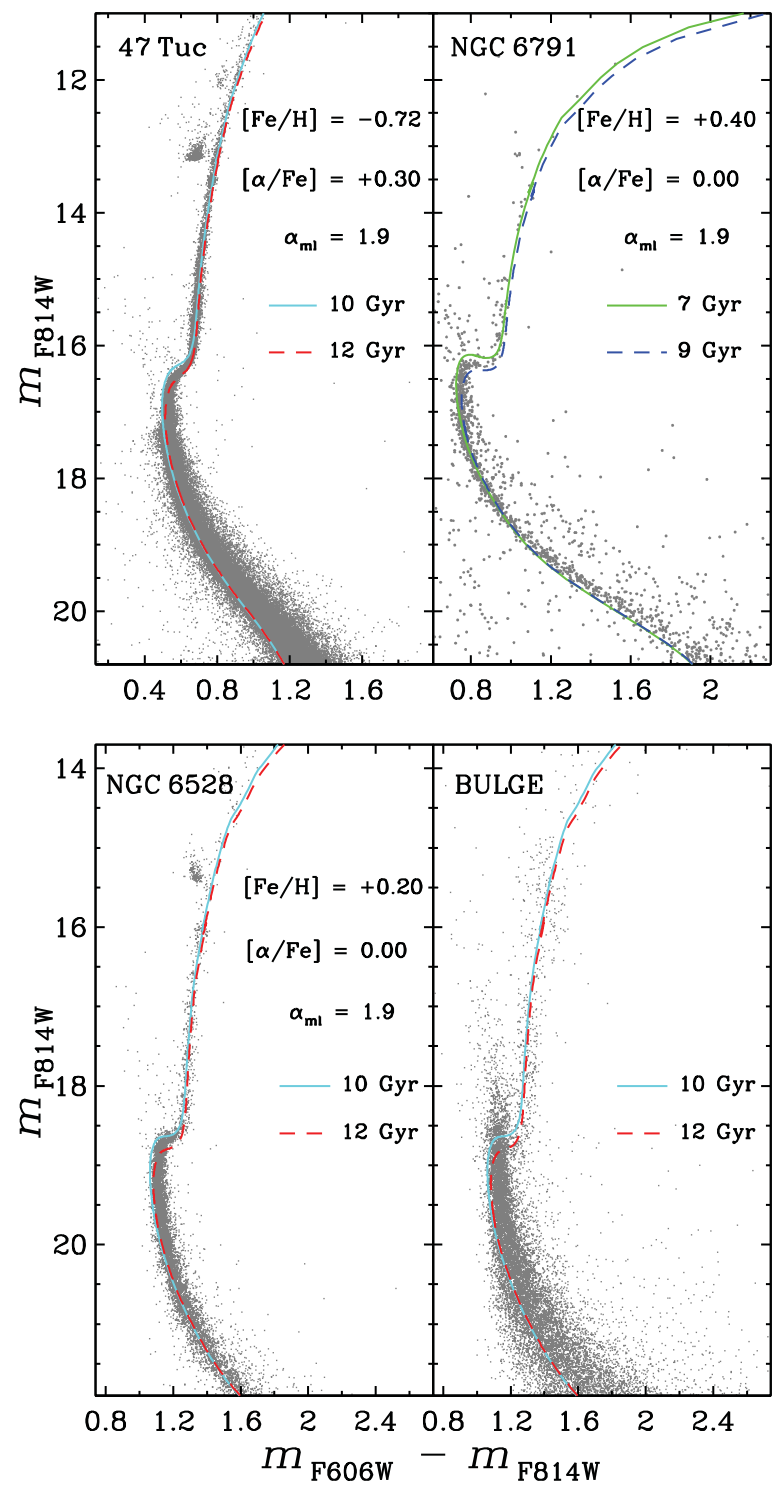

Figure 12. Top left: comparison in the $m_{\mathrm{F} 814 \mathrm{~W}}, m_{\mathrm{F} 606 \mathrm{~W}}-m_{\mathrm{F} 814 \mathrm{~W}} \mathrm{CMD}$ between stellar isochrones and optical ACS photometry for the GGC 47 Tuc. The cluster isochrones range from 10 to $12 \mathrm{Gyr}$ and are based on a set of $\alpha$-enhanced evolutionary models constructed at fixed chemical composition (see the labeled values). The adopted true distance modulus and reddening are the same as in Figure 11. Top right: same as the left, but for the cluster NGC 6791. The isochrones range from 7 to 9 Gyr. They were constructed by assuming a scaledsolar mixture and a super-metal-rich chemical composition. Bottom left: same as the top, but for the GGC NGC 6528. The cluster isochrones range from 10 to 12 Gyr. They were constructed assuming a scaled-solar mixture and a super-metal-rich chemical composition. Bottom right: same as the left, but for the candidate bulge stars.

(A color version of this figure is available in the online journal.)

that NGC 6528 seems to be coeval with 47 Tuc, and indeed we found that its age is $11 \pm 1$ Gyr. Moreover, the shape of both the SGB and the RGB support the hypothesis that this globular has a super-metal-rich iron abundance. The cluster age agrees quite well with the most recent estimates available in the literature: $t \sim 13 \mathrm{Gyr}$, (Zoccali et al. 2001), $t=11 \pm 2$, (Feltzing \& Johnson 2002); $t \sim 12.6$ Gyr, (Momany et al. 2003); $t \sim 12.5 \mathrm{Gyr}$, (Brown et al. 2005).

The bottom right panel shows the comparison between the candidate bulge stars and the same set of old isochrones. A detailed analysis of the age and metallicity distribution of bulge stars is beyond the scope of the current investigation. However, data plotted in the bottom right panel display that old, super metal-rich isochrones account for a sizable sample of field RGs and a plausible fit for the bulk of SGB and MSTO stars. The comparison further supports the evidence for a spread both in age and in chemical composition.

We note that the adopted iron abundance for NGC 6528 , $[\mathrm{Fe} / \mathrm{H}]=+0.2$ is slightly higher than current spectroscopic measurements $([\mathrm{Fe} / \mathrm{H}]=-0.1 \pm 0.2$, Zoccali et al. 2004; $[\mathrm{Fe} / \mathrm{H}]=-0.17 \pm 0.01$ Origlia et al.; 2005; $[\mathrm{Fe} / \mathrm{H}]=$ $+0.07 \pm 0.08$ Carretta et al. 2001). No firm conclusion can be reached concerning the abundance of $\alpha$ elements. The fit with scaled-solar isochrones is slightly better than with $\alpha$-enhanced isochrones. The same outcome applies to spectroscopic observations, and indeed, Zoccali et al. (2004) found $[\alpha / \mathrm{Fe}] \approx+0.1$, while Origlia et al. (2005) and Carretta et al. (2001) found $[\alpha / \mathrm{Fe}] \approx+0.3$. Independent constraints will require accurate and deep NIR photometry and larger samples of high-resolution spectra.

\section{SUMMARY AND FINAL REMARKS}

On the basis of a large set of optical and near-infrared images collected with ACS/WFC, WFC3/UVIS, and WFC3/IR on board the $H S T$, we performed deep and precise photometry of the Galactic globular NGC 6528. Current data set covers a time interval of almost $10 \mathrm{yr}$ and allowed us to perform accurate PM measurements over the entire FOV of the above images. The PMs were used to separate candidate field and cluster stars.

Moreover, we also performed detailed estimates of the reddening map over the entire FOV of the above images and provided reddening corrections for individual objects. The PM selection and the correction for differential reddening brought forward very accurate and deep CMDs for both field and cluster stars. This applies not only to evolved evolutionary features (red HB, red clump, RGB bump), but also to MS stars fainter than the MSTO.

The comparison with empirical calibrators (47 Tuc, NGC 6791) and with cluster isochrones indicate that NGC 6528 is an old GGC with super-solar iron abundance. Together with 47 Tuc, it seems to belong to the group of old GGCs that are characterized by a minimal dispersion in age and no evidence of an age-metallicity relation (Marín-Franch et al. 2009). The above findings together with the solid empirical evidence of an overabundance of $\alpha$-elements in field bulge stars over the entire metallicity range (McWilliam \& Rich 1994; Zoccali et al. 2006; Gonzalez et al. 2011; Ness et al. 2013a, 2013b) further support the hypothesis that the high rate of star formation in the innermost regions of the bulge caused, in the first $\sim 1$ Gyr, a fast chemical enrichment of the interstellar medium.

Our findings concerning NGC 6528 support previous results from different authors on a rapid chemical enrichment of the bulge. In particular, a thorough high-spectral-resolution spectroscopic survey of the Galactic bulge (ARGOS) was recently performed by Freeman et al. (2013) and Ness et al. (2013a, 2013b). On the basis of the metallicity distribution function and of the kinematic properties of a large sample of RC stars they found that the metal-rich stellar component $([\mathrm{Fe} / \mathrm{H}]>-0.5)$ is associated with the boxy/peanut (McWilliam \& Zoccali 2010) bulge (components A, B), while the metal-poor component $([\mathrm{Fe} / \mathrm{H}]<-0.5)$ is associated with both the Galactic metal-intermediate thick disc and the metal-poor halo (components $\mathrm{C}, \mathrm{D}, \mathrm{E})$. The bulge stellar components include two spatially and chemically distinct sub-populations: (1) a thin, 
more metal-rich sub-sample $([\mathrm{Fe} / \mathrm{H}] \approx 0.15)$ that is kinematically colder and closer to the Galactic plane. This component is associated to the thin disc of the Galaxy (A component). (2) A thick, more metal-poor sub-sample $([\mathrm{Fe} / \mathrm{H}] \approx-0.25)$ that is uniformly distributed across the selected fields. This component is associated to the boxy/peanut bulge and it is considered the tracer of the pristine bulge stellar population. It is also characterized by a large vertical distribution formed via an early disk instability that dragged the first generation of the thin disc stars into the boxy/peanut bulge structure (Ness et al. 2013a, 2013b).

It goes without saying that current empirical scenario concerning the metallicity distribution of bulge stars awaits for a comprehensive analysis of the iron distribution and of the $\alpha$ element distribution of stellar tracers tightly connected with the pristine stellar population of the bulge, namely blue/red HB stars and RR Lyræ stars.

It is a pleasure to thank an anonymous referee for pertinent suggestions and comments that improved the content and the readability of the manuscript. This work was partially supported by PRIN INAF 2011 "Tracing the formation and evolution of the Galactic halo with VST" (PI: M. Marconi) and by PRIN-MIUR (2010LY5N2T) "Chemical and dynamical evolution of the Milky Way and Local Group galaxies" (PI: F. Matteucci). A.P.M. acknowledges the financial support from the Australian Research Council through Discovery Project grant DP120100475. Support for this work has been provided by the IAC (grant 310394), and the Education and Science Ministry of Spain (grants AYA2007-3E3506, and AYA2010-16717).

\section{REFERENCES}

Anderson, J., \& King, I. R. 2003, AJ, 126, 772

Anderson, J., \& King, I. R. 2006, Instrument Science Report ACS 2006-01, 34 pages, 1

Anderson, J., Sarajedini, A., Bedin, L. R., et al. 2008, AJ, 135, 2055

Asplund, M., Grevesse, N., Sauval, A. J., \& Scott, P. 2009, ARA\&A, 47, 481

Baade, W. 1958, in Stellar Population, ed. D. J. K. O'Connell (New York: Interscience), 303

Bedin, L. R., Anderson, J., King, I. R., \& Piotto, G. 2001, ApJL, 560, L75

Bedin, L. R., Cassisi, S., Castelli, F., et al. 2005, MNRAS, 357, 1038

Bellini, A., Anderson, J., \& Bedin, L. R. 2011, PASP, 123, 622

Bergbusch, P. A., \& Stetson, P. B. 2009, AJ, 138, 1455

Bertelli, G., Bressan, A., Chiosi, C., Fagotto, F., \& Nasi, E. 1994, A\&AS, 106,275

Boesgaard, A. M., Jensen, E. E. C., \& Deliyannis, C. P. 2009, AJ, 137, 4949

Bono, G., Stetson, P. B., VandenBerg, D. A., et al. 2010, ApJL, 708, L74

Brasseur, C. M., Stetson, P. B., VandenBerg, D. A., et al. 2010, AJ, 140, 1672

Brogaard, K., VandenBerg, D. A., Bruntt, H., et al. 2012, A\&A, 543, A106
Brott, I., \& Hauschildt, P. H. 2005, in The Three-Dimensional Universe with Gaia, ed. C. Turon, K. S. O'Flaherty, \& M. A. C. Perryman (ESA SP-576; Noordwijk: ESA), 565

Brown, T. M., Ferguson, H. C., Smith, E., et al. 2005, AJ, 130, 1693

Calamida, A., Bono, G., Stetson, P. B., et al. 2009, ApJ, 706, 1277

Calamida, A., Monelli, M., Milone, A. P., et al. 2012, A\&A, 544, A152

Carretta, E., Bragaglia, A., Gratton, R., D’Orazi, V., \& Lucatello, S. 2009, A\&A, 508,695

Carretta, E., Cohen, J. G., Gratton, R. G., \& Behr, B. B. 2001, AJ, 122, 1469

Cassisi, S., \& Salaris, M. 1997, MNRAS, 285, 593

Castellani, V., Degli' Innocenti, S., Fiorentini, G., Lissia, M., \& Ricci, B. 1999 $\mathrm{PhR}, 281,309$

Castelli, F., \& Kurucz, R. L. 2003, in IAU Symp. 210, Modelling of Stellar Atmospheres, ed. N. Piskunov, W. W. Weiss, \& D. F. Gray (Cambridge: Cambridge Univ. Press), 20

Degl'Innocenti, S., Prada Moroni, P. G., Marconi, M., \& Ruoppo, A 2008, Ap\&SS, 316, 25

Dell'Omodarme, M., Valle, G., Degl'Innocenti, S., \& Prada Moroni, P. G. 2012, A\&A, 540, A26

Dotter, A., Sarajedini, A., \& Anderson, J. 2011, ApJ, 738, 74

Dotter, A., Sarajedini, A., Anderson, J., et al. 2010, ApJ, 708, 698

Feltzing, S., \& Johnson, R. A. 2002, A\&A, 385, 67

Freeman, K., Ness, M., Wylie-de-Boer, E., et al. 2013, MNRAS, 428, 3660

Gennaro, M., Prada Moroni, P. G., \& Degl'Innocenti, S. 2010, A\&A, 518, A13

Gonzalez, O. A., Rejkuba, M., Zoccali, M., et al. 2011, A\&A, 530, A54

Hansen, B. M. S., Kalirai, J. S., Anderson, J., et al. 2013, Natur, 500, 51

Harris, W. E. 1996, AJ, 112, 1487

Jimenez, R., Flynn, C., MacDonald, J., \& Gibson, B. K. 2003, Sci, 299, 1552

Kuijken, K., \& Rich, R. M. 2002, AJ, 124, 2054

Marín-Franch, A., Aparicio, A., Piotto, G., et al. 2009, ApJ, 694, 1498

McLaughlin, D. E., Anderson, J., Meylan, G., et al. 2006, ApJS, 166, 249

McWilliam, A., \& Rich, R. M. 1994, ApJS, 91, 749

McWilliam, A., \& Zoccali, M. 2010, ApJ, 724, 1491

Milone, A. P., Bedin, L. R., Piotto, G., \& Anderson, J. 2009, A\&A, 497, 755

Milone, A. P., Piotto, G., Bedin, L. R., et al. 2012, A\&A, 540, A16

Momany, Y., Ortolani, S., Held, E. V., et al. 2003, A\&A, 402, 607

Ness, M., Freeman, K., Athanassoula, E., et al. 2013a, MNRAS, 430, 836

Ness, M., Freeman, K., Athanassoula, E., et al. 2013b, MNRAS, 432, 2092

Origlia, L., Valenti, E., \& Rich, R. M. 2005, MNRAS, 356, 1276

Ortolani, S., Barbuy, B., Bica, E., et al. 2001, A\&A, 376, 878

Ortolani, S., Renzini, A., Gilmozzi, R., et al. 1995, Natur, 377, 701

Pagel, B. E. J., \& Portinari, L. 1998, MNRAS, 298, 747

Peimbert, M., Luridiana, V., \& Peimbert, A. 2007, ApJ, 666, 636

Rakos, K., \& Schombert, J. 2005, PASP, 117, 245

Salaris, M., Held, E. V., Ortolani, S., Gullieuszik, M., \& Momany, Y. 2007, A\&A, 476, 243

Salasnich, B., Girardi, L., Weiss, A., \& Chiosi, C. 2000, A\&A, 361, 1023

Steigman, G. 2006, IJMPE, 15, 1

VandenBerg, D. A., Brogaard, K., Leaman, R., \& Casagrande, L. 2013, ApJ, 775,134

VandenBerg, D. A., Casagrande, L., \& Stetson, P. B. 2010, AJ, 140, 1020 van Tulder, J. J. M. 1942, BAN, 9, 315

Zoccali, M., Barbuy, B., Hill, V., et al. 2004, A\&A, 423, 507

Zoccali, M., Lecureur, A., Barbuy, B., et al. 2006, A\&A, 457, L1

Zoccali, M., Renzini, A., Ortolani, S., Bica, E., \& Barbuy, B. 2001, AJ, 121,2638 


\title{
ERRATUM: “ON THE KINEMATIC SEPARATION OF FIELD AND CLUSTER STARS ACROSS THE BULGE GLOBULAR NGC 6528” (2014, ApJ, 782, 50)
}

\author{
E. P. Lagioia ${ }^{1}$, A. P. Milone ${ }^{2,3,4}$, P. B. Stetson ${ }^{5}$, G. Bono ${ }^{1,6}$, P. G. Prada Moroni ${ }^{7}$, M. Dall'ora ${ }^{8}$, \\ A. Aparicio ${ }^{3,4}$, R. Buonanno ${ }^{1,9}$, A. Calamida ${ }^{6,10}$, I. Ferraro $^{6}$, R. Gilmozzi $^{11}$, G. IAnNicola $^{6}$, \\ N. MatsunaGa ${ }^{12}$, M. Monelli ${ }^{3,4}$, AND A. WALKER ${ }^{13}$ \\ ${ }^{1}$ Dipartimento di Fisica, Università degli Studi di Roma-Tor Vergata, via della Ricerca Scientifica 1, I-00133 Roma, Italy; eplagioia@ roma2.infn.it \\ ${ }^{2}$ Research School of Astronomy and Astrophysics, The Australian National University, Cotter Road, Weston, ACT 2611, Australia \\ ${ }^{3}$ Instituto de Astrofisica de Canarias, E-38200 La Laguna, Tenerife, Canary Islands, Spain \\ ${ }^{4}$ Department of Astrophysics, University of La Laguna, E-38200 La Laguna, Tenerife, Canary Islands, Spain \\ ${ }^{5}$ Dominion Astrophysical Observatory, Herzberg Institute of Astrophysics, National Research Council, 5071 West Saanich Road, Victoria, BC V9E 2E7, Canada \\ ${ }^{6}$ INAF-Osservatorio Astronomico di Roma, Via Frascati 33, I-00044 Monte Porzio Catone, Italy \\ ${ }^{7}$ Dipartimento di Fisica, Università di Pisa, I-56127 Pisa, Italy \\ ${ }^{8}$ INAF-Osservatorio Astronomico di Capodimonte, Salita Moiariello 16, I-80131 Napoli, Italy \\ ${ }^{9}$ INAF-Osservatorio Astronomico Collurania, Via M. Maggini, I-64100 Teramo, Italy \\ ${ }^{10}$ Space Telescope Science Institute, 3700 San Martin Drive, Baltimore, MD 21218, USA \\ ${ }^{11}$ European Southern Observatory, Karl-Schwarzschild-Straße 2, D-85748 Garching, Germany \\ 12 Kiso Observatory, Institute of Astronomy, School of Science, The University of Tokyo, 10762-30, Mitake, Kiso-machi, Kiso-gun, 3 Nagano 97-0101, Japan \\ ${ }^{13}$ Cerro Tololo Inter-American Observatory, National Optical Astronomy Observatory, Casilla 603, La Serena, Chile \\ Received 2014 March 5; published 2014 March 27
}

Online-only material: color figures

Due to an error at the publisher, there were several errors in the published version of this paper. The sign of the proper motions along the $Y$-axis of Figures 3-5, 8, and 9 was incorrect and the corrected versions of these figures are reproduced below.
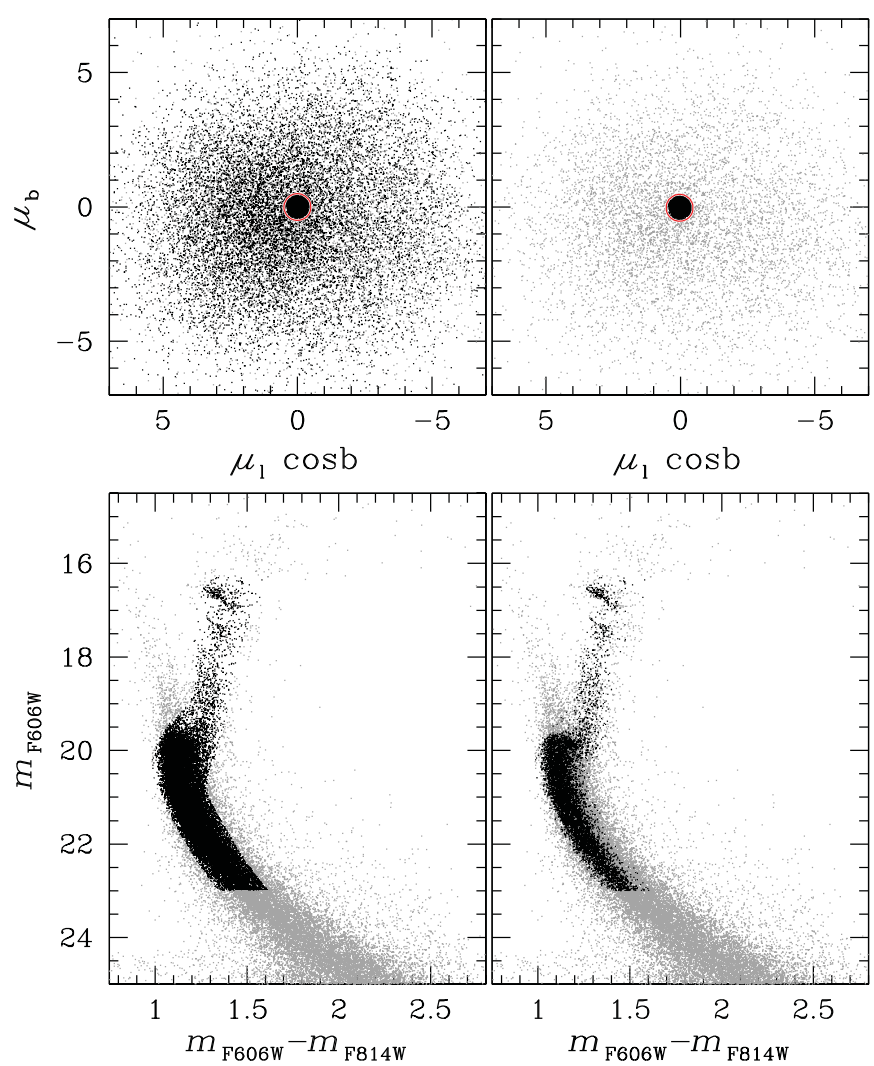

Figure 3. Proper motion of stars in the field of view of NGC 6528. The stars used as reference to measure proper motions are marked as black points in the CMDs (bottom panels) and in the vector-point diagram (top panels). The left panels show the sample of reference stars selected only on the basis of their position in the $\mathrm{CMD}$ and used to obtain a first preliminary estimate of proper motions. The right panels display the sample of reference stars used for the final measurement of proper motions and selected on the basis of their position both in the CMD and in the vector-point diagram.

(A color version of this figure is available in the online journal.) 


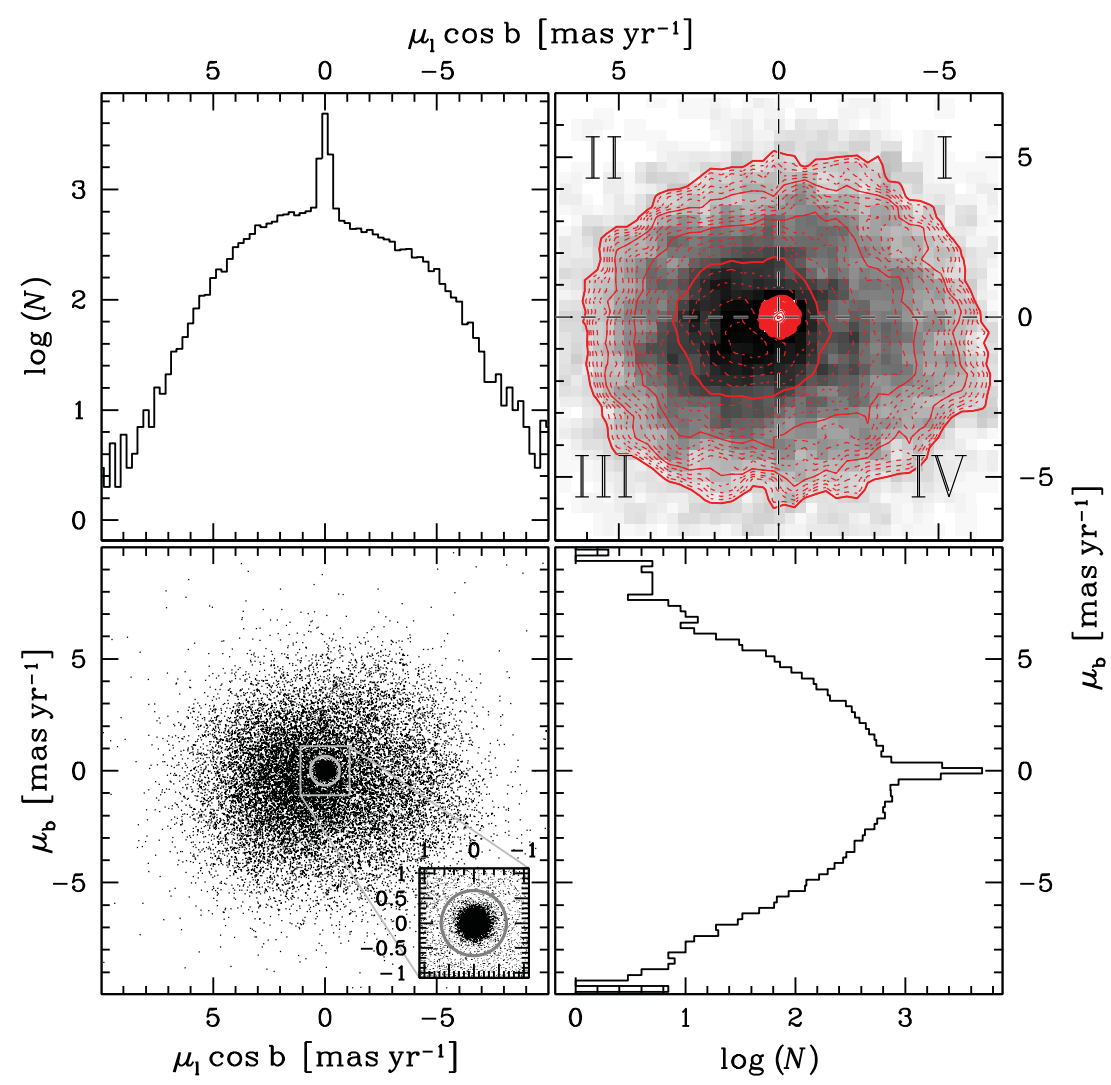

Figure 4. Bottom left: VPD of the proper-motion distribution of the entire sample of stars: for the sake of clarity only stars with $m_{\mathrm{F} 814 \mathrm{~W}}<22.1$ have been plotted across the field of view of NGC 6528. The inset shows a zoom-in around the origin of the VPD. The bulk of stars around $\left(\mu_{1} \cos b, \mu_{\mathrm{b}}\right)=(0,0)$ mainly consists of cluster members. Top right: smoothed VPD diagram and contours of the previous sample. The dashed lines separate the four quadrants. The top-left and the bottom-right panels show the marginals of the proper motions along the $\mu_{1} \cos b$, and the $\mu_{\mathrm{b}}$ axis.

(A color version of this figure is available in the online journal.)
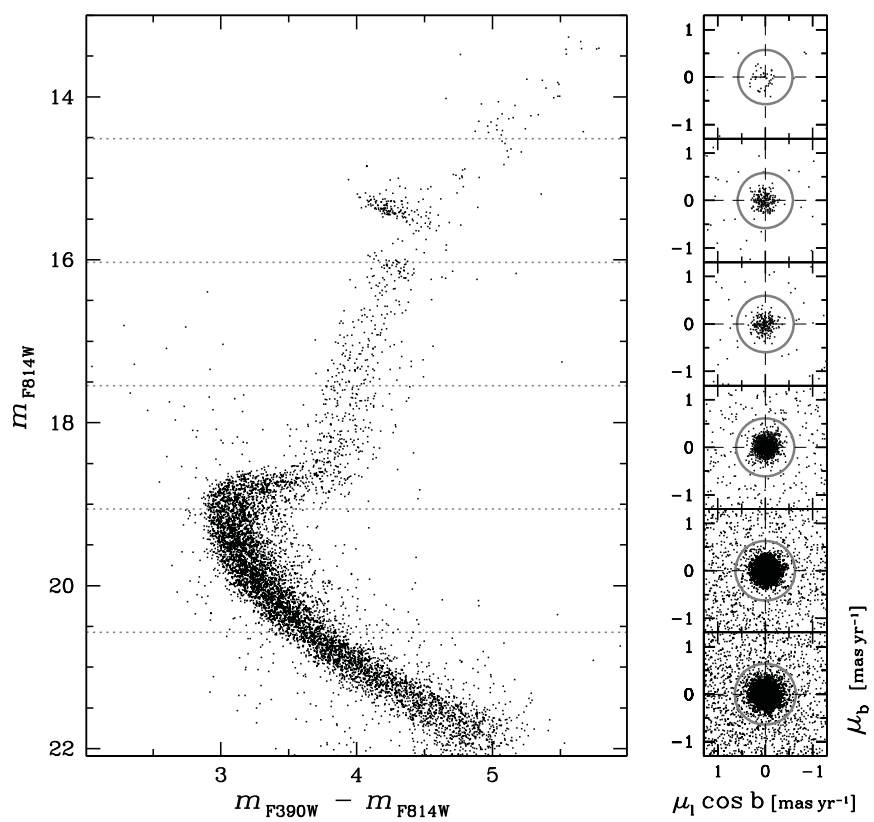

Figure 5. Left: $m_{\mathrm{F} 814 \mathrm{~W}}, m_{\mathrm{F} 390 \mathrm{~W}}-m_{\mathrm{F} 814 \mathrm{~W}} \mathrm{CMD}$ of candidate cluster stars. Right: VPD for the stars in 6 mag bins, marked by dotted lines in the left panel. Gray circle separate candidate cluster members from field stars (see text for details).

Furthermore, there are several places in the text that should be changed as well. They are as follows.

1. The third row of the right column on page 4 should read, "in the third quadrant as indicated by the contours."

2. The seventh row of the right column on page 4 should be changed to "slopes at positive Galactic longitudes and negative latitudes are steeper than at negative and positive ones, respectively. The mode of the field-star VPD also seems to be displaced toward positive longitudes and negative latitudes, relative to." 


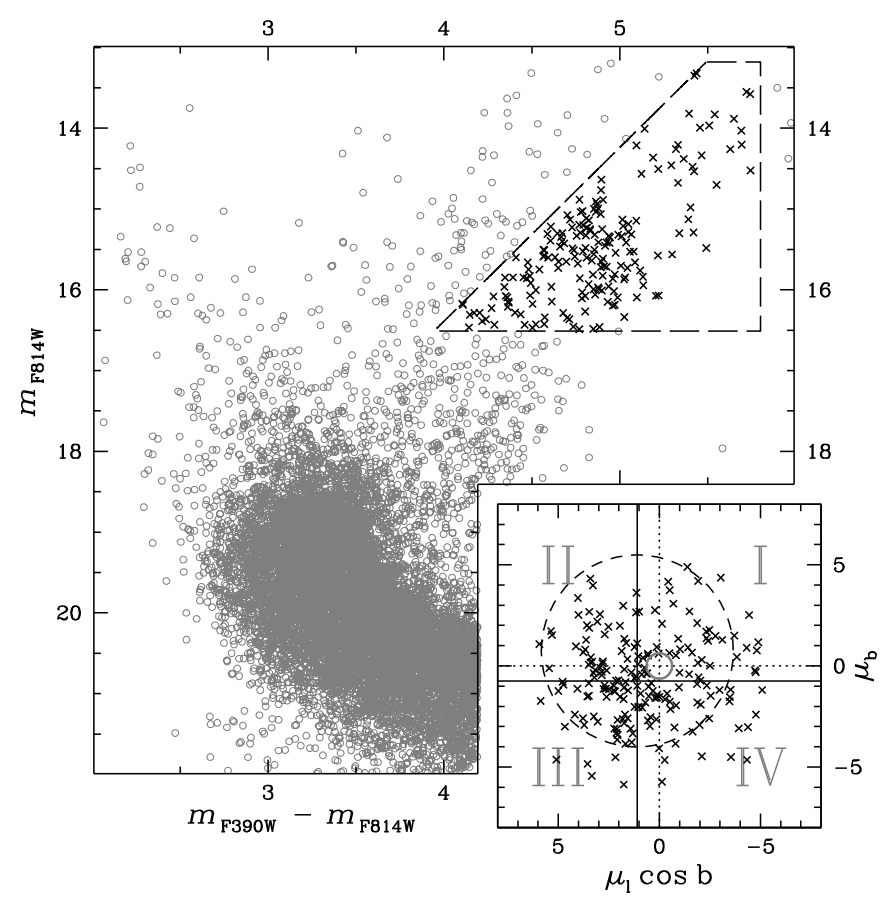

Figure 8. $m_{\mathrm{F} 814 \mathrm{~W}}, m_{\mathrm{F} 390 \mathrm{~W}}-m_{\mathrm{F} 814 \mathrm{~W}} \mathrm{CMD}$ of field stars. The black crosses located inside the dashed window, mark stars that, according to their position in the CMD, are probable bulge members. The position of these stars in the VPD is shown in the inset.
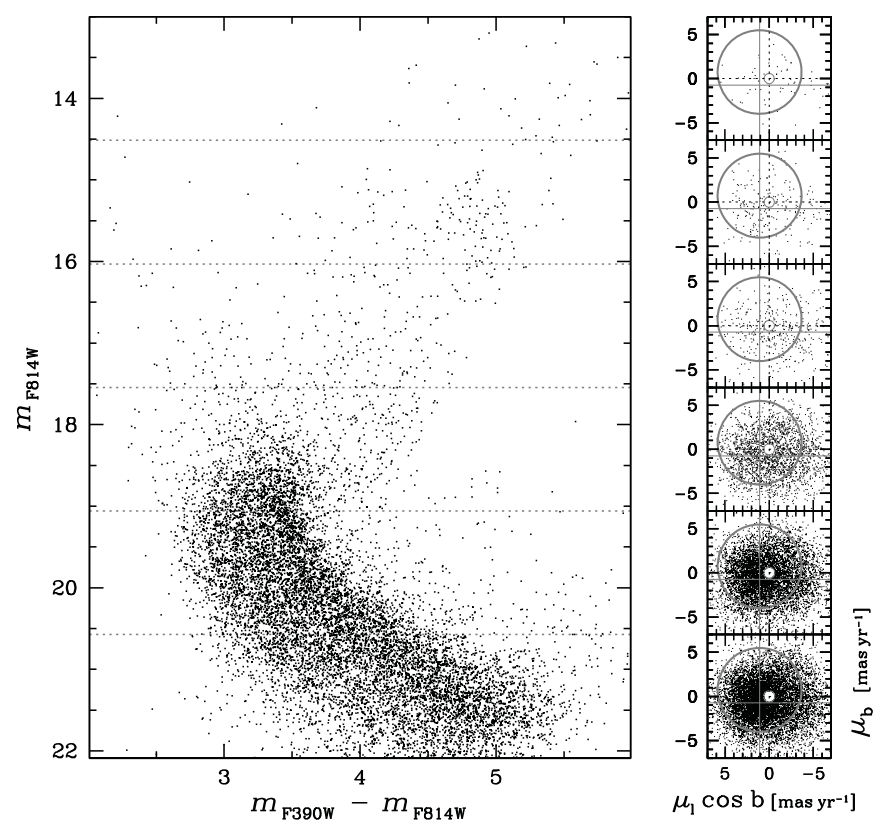

Figure 9. Left panel: $m_{\mathrm{F} 814 \mathrm{~W}}$ vs. $m_{\mathrm{F} 390 \mathrm{~W}}-m_{\mathrm{F} 814 \mathrm{~W}} \mathrm{CMD}$ for candidate bulge stars. Right panels: VPD of field stars in 6 mag bins. Gray circles separate candidate bulge stars from remaining field stars (see text for details).

3. In the seventeenth row of Section 3, "+0.74" should be " $-0.74 . "$

4. In the 20th row of Section 3, "quadrant II" should be "quadrant III."

The above changes do not affect any of the conclusions of the paper.

IOP Publishing sincerely regrets these errors. 